\title{
Distributed algorithms for sum rate maximization in multi-cell downlink OFDMA with opportunistic DF relaying
}

\author{
Zhiwen Jin ${ }^{1 *}$, Tao Wang ${ }^{2}$, Jibo Wei ${ }^{3}$ and Luc Vandendorpe ${ }^{1}$
}

\begin{abstract}
This paper considers a multi-cell orthogonal frequency division multiple access (OFDMA) downlink system with several decode-and-forward (DF) relay stations (RSs) aiding the base station (BS) transmissions. The problem considered is the maximization of the system sum rate with a total power constraint in each cell. An iterative semi-distributed resource allocation (RA) algorithm is first proposed to optimize mode selection (decision whether relaying should be used or not and which relay), subcarrier assignment (MSSA), and power allocation (PA), alternatively. During the MSSA stage, the problem is decoupled into subproblems which can be solved distributively in linear time. During the PA stage, an algorithm based on single condensation and Lagrange duality (SCLD) is designed to optimize PA with the tentative MSSA results. The convergence of the SCLD-based RA algorithm is theoretically guaranteed and an local optimum is reached after convergence. To solve the formulated problem autonomously, a modified iterative water-filling (IWF) algorithm is further proposed. Specifically, each cell autonomously optimizes its own sum rate with the estimated power values of the received interferences from the other cells. An optimum algorithm is proposed to solve the local RA problem in each cell. Through numerical experiments, the convergence of the two proposed algorithms as well as their benefits compared with a centralized algorithm (CA) are illustrated.
\end{abstract}

Keywords: OFDMA; Opportunistic DF relaying; Distributed resource allocation; Cochannel interference mitigation

\section{Introduction}

High data rate and ubiquitous coverage are strongly required in the next-generation wireless communication networks. To achieve this goal, the emerging technology incorporating orthogonal frequency division multiple access (OFDMA) with relaying is receiving a lot of interest from both academia and industry. In particular, the OFDMA technology is able to combat frequencyselective multi-path fading and flexible in applying dynamic radio resource allocation (RA) for performance improvement. Moreover, the relaying technology has the attractive feature of coverage extension and data rate improvement [1].

Concerning relay-aided OFDM(A) transmissions, [2] and [3] have proposed two types of relaying, namely

\footnotetext{
*Correspondence: zhiwen.jin@student.uclouvain.be

1 ICTEAM institute (École Polytechnique de Louvain), Université catholique de

Louvain, Place du Levant, 1348 Louvain-la-Neuve, Belgium

Full list of author information is available at the end of the article
}

amplify-and-forward (AF) and decode-and-forward (DF) relays. With DF/AF relaying, symbol transmission is carried out in two time slots (TSs). During the first TS, the source broadcasts symbols on all subcarriers with the relay keeping quiet. During the second TS, except for the relay, the source might also transmit symbols on subcarriers unused by the relay. Adopting the DF relaying, [4-23] have investigated intensively the RA problems for downlink OFDMA.

Considering the case when the source to destination (S-D) link is unavailable, [4-7] have proposed algorithms for RA in the DF relay-aided OFDM systems. Considering the case when the S-D link is available, [8-23] have studied RA in systems with opportunistic relaying (sometimes termed as selection relaying). To start with, [8-13] have studied a low spectrum efficiency (LSE) protocol, when only the relay transmits symbols during the second TS. Specifically, each subcarrier can choose either the relayaided mode or the direct mode for data transmission. In

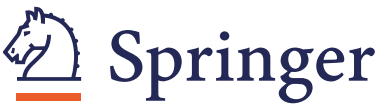

(c) 2014 Jin et al: licensee Springer. This is an Open Access article distributed under the terms of the Creative Commons

Attribution License (http://creativecommons.org/licenses/by/4.0), which permits unrestricted use, distribution, and reproduction in any medium, provided the original work is properly credited. 
the relay-aided mode, a symbol is first transmitted from the source to the relay during the first TS, which is then forwarded from the relay to the destination during the second TS. While in the direct mode, the symbol is transmitted directly to the targeted destination during the first TS without the help of the relay. Note that, with the LSE protocol, subcarriers unused by the relay during the second TS are actually wasted. To address this issue, [14-22] have proposed and studied improved high spectrum efficiency (HSE) protocols which allow new symbols to be transmitted on the subcarriers unused by the relay during the second TS.

Note that all these papers consider RA in single-cell situations and model the cochannel interference (CCI) as additive background noise. This is reasonable only when the frequency reuse factor $1 / W$ is low. Here, $W$ is the number of cells in a cluster which cannot use the same frequencies for transmission. However, due to its ability to achieve higher system capacity, aggressive frequency reuse is recommended in the next generation cellular systems [24]. When the frequency reuse factor is high, the CCI becomes a key factor affecting the system performance and thus cannot be ignored $[25,26]$.

Considering the CCI, system models in multi-cell DF relay-aided OFDMA become quite interesting and challenging. With multi-cell DF relay-aided OFDMA systems, [27] and [28] have discussed RA algorithms when the powers are uniformly allocated to all stations. For multicell OFDMA systems without DF relaying, several RA algorithms have been proposed in [29-32]. However, these methods cannot be extended directly to solve RA problems jointly optimizing transmission mode selection, subcarrier assignment (MSSA), as well as power allocation (PA) in multi-cell OFDMA systems with opportunistic DF relaying. Concerning the opportunistic DF protocol in multi-cell relayed OFDMA systems, [25] and [26] have recently proposed joint RA schemes to maximize the sum rate over all cells and the weighted sum of each cell min-rate, respectively. However, the proposed centralized algorithms (CAs) seem to be quite heavy to implement in practice.

Compared with the above existing works, the contributions of this paper are as follows:

- We propose an iterative semi-distributed RA algorithm to optimize the MSSA and the PA alternatively with the sum rate keeping increasing. For the MSSA stage, the optimization problem is decoupled into subproblems which can be solved distributively in linear time. For the PA stage, the algorithm based on single condensation and Lagrange duality (SCLD) is designed to optimize PA iteratively with an analytical solution at each iteration. As will be illustrated through numerical results, the proposed algorithm converges quite fast and is more practical compared to the CA of [25].

- We further propose a modified iterative water-filling (IWF) algorithm to solve the formulated RA problem autonomously. Specifically, each cell autonomously optimizes its own sum rate with the estimated power values of the received interferences from the other cells. An optimum algorithm is proposed to solve the local RA problem in each cell. As will be illustrated, the convergence of the modified IWF algorithm is always observed in simulations, although it seems intractable to derive the conditions of convergence theoretically. Through numerical results, the modified IWF algorithm provides a good trade-off between complexity and performance. Thus, it is recommended for practical implementation.

The rest of this paper is organized as follows: First, the system model and the problem formulation for the considered system are presented in the next section. The proposed SCLD-based RA algorithm and the modified IWF algorithm are described in 'Section 3' and 'Section 4', respectively. The convergence of the proposed algorithms and their benefits compared with a CA are illustrated by numerical experiments in 'Section 5'. Finally, the conclusions are drawn in 'Section 6'.

\section{System model and problem formulation 2.1 System model}

We consider a cellular OFDMA system with $N$ cells coordinated by a central controller for message passing among cells. In each cell, downlink transmission is carried out from a base station (BS) to $U$ mobile stations (MSs) with the help of $J$ relay stations (RSs) which are assumed to be of the DF type. For each link, the channel is assumed to be frequency selective and transformed into $K$ parallel subchannels by using OFDM with sufficiently long cyclic prefix. The data transmission is carried out in two TSs. During the first TS, a symbol is first broadcast by the $\mathrm{BS}$ at a subcarrier $k$, which is in either relay-aided mode or direct mode. Both a selected RS and a targeted MS receive this symbol. If the relay-aided mode is used, the RS decodes the received symbol and forwards it to the targeted MS over the subcarrier $k$ with the BS keeping quiet on this subcarrier during the second TS. The MS only decodes the symbol received during the second TS. If the direct mode is used, the targeted MS decodes the symbol received during the first TS. Also, another symbol is broadcast by the BS at the same subcarrier during the second TS, which is received and only decoded by the targeted MS. 
With OFDMA, each subcarrier is allocated to only one MS in each cell. Throughout this paper, we assume the timing and carrier synchronization is perfect and there are no errors during the SR transmission at the first TS. We further assume that the central controller can obtain/distribute messages from/to all nodes. Moreover, we assume that the coherence time of each link is sufficiently long for implementing the RA. Note that by assuming the above idealities, an upper bound on the system performances is obtained.

The data transmission procedure in every cell is identical. Thus, we only analyze the downlink data transmission inside one selected cell $n$, which is impaired by cochannel interference from the other cells. Specifically in cell $n$, data transmissions are carried out either in relay-aided mode or direct mode, as will be elaborated in the following section. Note that, as will be used, a variable with the indice $t 1 / t 2$ means that this variable corresponds to the transmission at TS1/TS2.

Let us first see more closely the relay-aided data transmission. As illustrated in Figure 1a, the BS $s_{n}$ first produces a symbol $\sqrt{P_{\mathrm{S}_{n}, t_{1}}^{k}} x_{\mathrm{s}_{n}, t_{1}}^{k}$ at subcarrier $k$ during the first TS, while the transmitter of the selected RS $r_{j n}$ remains idle. Here, $x_{\mathbf{s}_{n}, t_{1}}^{k}$ denotes the normalized symbol (meaning $E\left\{\left|x_{\mathrm{s}_{n}, t_{1}}^{k}\right|^{2}\right\}=1$ ) transmitted by $\mathrm{s}_{n}$ at subcarrier $k$ during the first TS, and $P_{\mathrm{s}_{n}, t_{1}}^{k}$ denotes the corresponding transmit power. Simultaneously in an interfering cell $n^{\prime}$, a symbol $\sqrt{P_{\mathrm{s}_{n^{\prime}}, t_{1}}^{k}} x_{\mathrm{s}_{n^{\prime}}, t_{1}}^{k}$ is also produced from the interfering BS $s_{n^{\prime}}$ at the same subcarrier. Here again $E\left\{\left|x_{s_{n^{\prime}}, t_{1}}^{k}\right|^{2}\right\}=1$. Note that, instead of using an additional integer variable to indicate whether $s_{n^{\prime}}$ transmits data on subcarrier $k$ or not, we use $\sqrt{P_{\mathrm{s}_{n^{\prime}}, t_{1}}^{k}}$ to do it. Specifically, $P_{\mathrm{s}_{n^{\prime}}, t_{1}}^{k}>0$ means that $\mathrm{s}_{n^{\prime}}$ uses subcarrier $k$ for data transmission during the first TS, and $P_{\mathrm{s}_{n^{\prime}}, t_{1}}^{k}=0$ means that $\mathrm{s}_{n^{\prime}}$ transmits nothing at the subcarrier $k$ during the first TS. This choice is motivated to simplify the system sum rate expression and facilitate the algorithm design. Specifically, when using an integer variable to indicate whether a subcarrier $k$ in a cell $n$ is used or not, the rate expression would contain integer variables in both the denominators and the numerators, which makes it a non-linear function of the integer variables. When using the corresponding power value to do it,

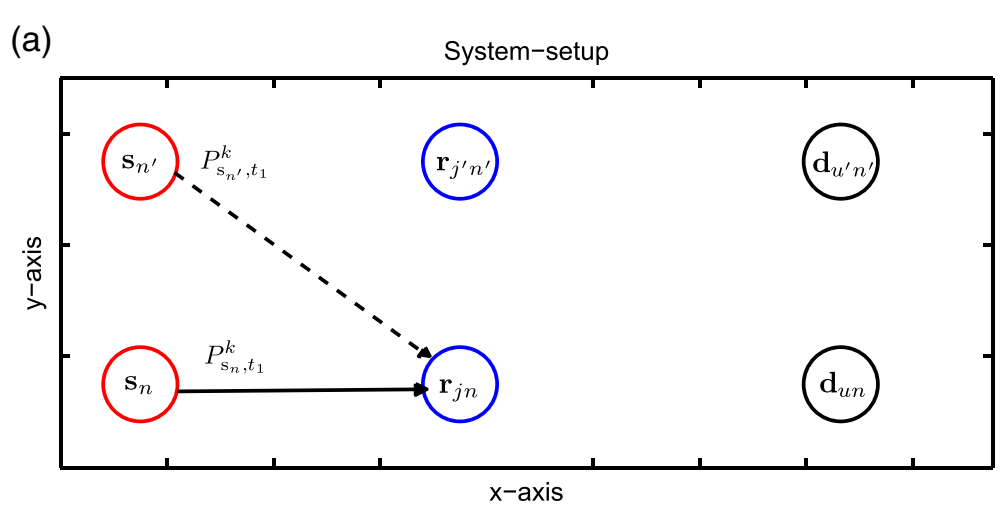

(b)

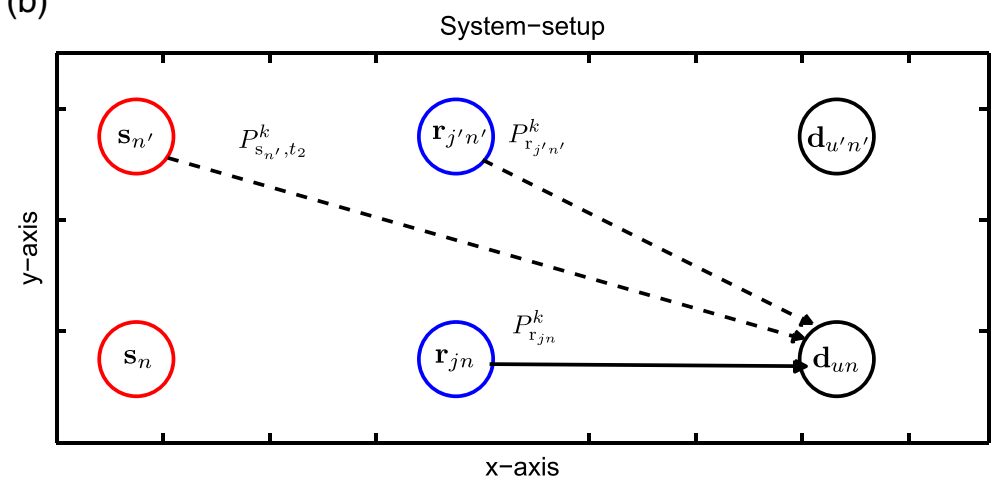

Figure 1 Illustration of a relay-aided transmission in cell $\boldsymbol{n}$ with cochannel interference from cell $\boldsymbol{n}^{\prime}$. The solid lines and the dashed lines represent communication links and interfering links, respectively. (a) At subcarrier $k$ and the first TS. (b) At subcarrier / and the second TS. 
the rate expression contains integer variables only in the numerators, which makes it a linear function of the integer variables. At the end of the first TS, the signal received by $\mathrm{r}_{j n}$ for subcarrier $k$ can be expressed as

$$
y_{\mathrm{r}_{j n}}^{k}=\sqrt{P_{\mathrm{s}_{n}, t_{1}}^{k}} h_{\mathrm{s}_{n}, \mathrm{r}_{j n}}^{k} x_{\mathrm{s}_{n}, t_{1}}^{k}+v_{\mathrm{r}_{j n}}^{k}+\sum_{n^{\prime}=1, n^{\prime} \neq n}^{N} \sqrt{P_{\mathrm{s}_{n^{\prime}}, t_{1}}^{k}} h_{\mathrm{s}_{n^{\prime}}, \mathrm{r}_{j n}}^{k} x_{\mathrm{s}_{n^{\prime}}, t_{1}}^{k}
$$

where $v_{\mathrm{r}_{j n}}^{k}$ denotes the additive white Gaussian noise (AWGN) at subcarrier $k$ and $r_{j n}$ during the first TS. $h_{\mathrm{s}_{n^{\prime}}, \mathrm{r}_{j n}}^{k}$ denotes the channel frequency response (CFR) for subcarrier $k$ from $s_{n^{\prime}}$ to $r_{j n}$.

During the second TS, as illustrated in Figure 1b, the selected RS $\mathrm{r}_{j n}$ re-encodes the decoded symbol and forwards $\sqrt{P_{\mathrm{r}_{j n}}^{k}} x_{\mathrm{r}_{j n}}^{k}$ at the same subcarrier $k$. Here, $x_{\mathrm{r}_{j n}}^{k}=$ $x_{\mathrm{s}_{n}, t_{1}}^{k}$. The BS $\mathrm{s}_{n}$ transmits nothing on this subcarrier, meaning that $P_{\mathrm{s}_{n}, t_{2}}^{k}=0$. Here, $P_{\mathrm{r}_{j n}}^{k}$ and $P_{\mathrm{s}_{n}, t_{2}}^{k}$ denote the transmit power allocated to $r_{j n}$ and $s_{n}$, respectively at subcarrier $k$ during the second TS. At the same time, in an interfering cell $n^{\prime}, \mathrm{r}_{j^{\prime} n^{\prime}}$ and $\mathrm{s}_{n^{\prime}}$ also transmit $\sqrt{P_{\mathrm{r}_{j^{\prime} n^{\prime}}}^{k}} x_{\mathrm{r}_{j^{\prime} n^{\prime}}^{k}}^{k}$ and $\sqrt{P_{\mathrm{s}_{n^{\prime}}, t_{2}}^{k}} x_{\mathrm{s}_{n^{\prime}}, t_{2}}^{k}$ at subcarrier $k$, where at most only one power value out of $\sqrt{P_{\mathrm{r}_{j^{\prime} n^{\prime}}}^{k}} x_{\mathrm{r}_{j^{\prime} n^{\prime}}}^{k}$ and $\sqrt{P_{\mathrm{s}_{n^{\prime}}, t_{2}}^{k}} x_{\mathrm{s}_{n^{\prime}}, t_{2}}^{k}$ can be non-zero. More specifically, when subcarrier $k$ of cell $n^{\prime}$ chooses the direct mode for data transmission, $P_{\mathrm{r}_{j^{\prime} n^{\prime}}}^{k}=0$. Otherwise, $P_{\mathrm{s}_{n^{\prime}}, t_{2}}^{k}=0$. Here again $E\left\{\left|x_{\mathrm{r}_{j^{\prime} n^{\prime}}}^{k}\right|^{2}\right\}=1$ and $E\left\{\left|x_{\mathrm{s}_{n^{\prime}}, t_{2}}^{k}\right|^{2}\right\}=1$. At the end of the second TS, the signal received by the targeted $\mathrm{MS} \mathrm{d}_{u n}$ at subcarrier $k$ can be expressed as

$$
\begin{aligned}
y_{\mathrm{d}_{u n}, t_{2}}^{k}= & \sqrt{P_{\mathrm{r}_{j n}}^{k}} h_{\mathrm{r}_{j n}, \mathrm{~d}_{u n}}^{k} x_{\mathrm{r}_{j n}}^{k}+v_{\mathrm{d}_{u n}, t_{2}}^{k} \\
& +\sum_{n^{\prime}=1, n^{\prime} \neq n}^{N} \sqrt{P_{\mathrm{s}_{n^{\prime}}, t_{2}}^{k}} h_{\mathrm{s}_{n^{\prime}}, \mathrm{d}_{u n}}^{k} x_{\mathrm{s}_{n^{\prime}}, t_{2}}^{k} \\
& +\sum_{n^{\prime}=1, n^{\prime} \neq n}^{N} \sum_{j^{\prime}=1}^{J} \sqrt{P_{\mathrm{r}_{j^{\prime} n^{\prime}}}^{k}} h_{\mathrm{r}_{j^{\prime} n^{\prime}}, \mathrm{d}_{u n}}^{k} x_{\mathrm{r}_{j^{\prime} n^{\prime}}}^{k}
\end{aligned}
$$

where $v_{\mathrm{d}_{u n}, t_{2}}^{k}$ denotes the AWGN corrupting $\mathrm{d}_{u n}$ at subcarrier $k$ during the second TS,$h_{\mathrm{r}_{j^{\prime} n^{\prime}}, \mathrm{d}_{u n}}^{k}$ denotes the CFR for subcarrier $k$ from $\mathrm{r}_{j^{\prime} n^{\prime}}$ to $\mathrm{d}_{u n} \cdot h_{\mathrm{s}_{n^{\prime}}, \mathrm{d}_{u n}}^{k}$ denotes the CFR of subcarrier $k$ from $\mathrm{s}_{n^{\prime}}$ to $\mathrm{d}_{u n}$.

We assume $\left\{v_{\mathrm{r}_{j n}}^{k}, v_{\mathrm{d}_{u n}, t_{2}}^{k}\right\}$ are independent zero-mean circular Gaussian random variables with the same variance $\sigma^{2}$. After some mathematical calculations, the signalto-interference-plus-noise ratio (SINR) associated with decoding $x_{\mathbf{s}_{n}, t_{1}}^{k}$ from $y_{\mathbf{r}_{j n}}^{k}$ at $\mathrm{r}_{j n}$ during the first TS is expressed by

$$
\Gamma_{\mathrm{r}_{j n}}^{k}=\frac{P_{\mathrm{s}_{n}, t_{1}}^{k} G_{\mathrm{s}_{n}, \mathrm{r}_{j n}}^{k}}{f_{\mathrm{r}_{j n}}^{k}}
$$

where $f_{\mathrm{r}_{j n}}^{k}=\sigma^{2}+\sum_{n^{\prime}, n^{\prime} \neq n} P_{\mathrm{s}_{n^{\prime}}, t_{1}}^{k} G_{\mathrm{s}_{n^{\prime}}, \mathrm{r}_{j n}}^{k}$ denotes the sum power of the AWGN and the interference received by $r_{j n}$ at subcarrier $k$ during the first TS. $G_{\mathrm{s}_{n}, \mathrm{r}_{j n}}^{k}=\left|h_{\mathrm{s}_{n}, \mathrm{r}_{j n}}^{k}\right|^{2}$ denotes the channel gain of subcarrier $k$ from $\mathrm{s}_{n}$ to $\mathrm{r}_{j n}$.

Also, the SINR associated with decoding $x_{\mathbf{r}_{j n}}^{k}$, which equals $x_{\mathrm{s}_{n}, t_{1}}^{k}$, from $y_{\mathrm{d}_{u n}, \mathrm{r}_{j n}}^{k}$ at $\mathrm{d}_{u n}$ during the second TS is expressed by

$$
\Gamma_{\mathrm{d}_{u n}, t_{2}}^{k}=\frac{P_{\mathrm{r}_{j n}}^{k} G_{\mathrm{r}_{j n}, \mathrm{~d}_{u n}}^{k}}{f_{\mathrm{d}_{u n}, t_{2}}^{k}}
$$

where $f_{\mathrm{d}_{u n}, t_{2}}^{k}=\sigma^{2}+\sum_{n^{\prime}=1, n^{\prime} \neq n}^{N} P_{\mathrm{s}_{n^{\prime}}, t_{2}}^{k} G_{\mathrm{s}_{n^{\prime}}, \mathrm{d}_{u n}}^{k}+\sum_{n^{\prime}=1, n^{\prime} \neq n}^{N} \sum_{j^{\prime}=1}^{J} P_{\mathrm{r}_{j^{\prime} n^{\prime}}}^{k} G_{\mathrm{r}_{j^{\prime} n^{\prime}}, \mathrm{d}_{u n}}^{k}$ denotes the sum power of the AWGN and the interference received by $\mathrm{d}_{u n}$ at subcarrier $k$ during the second TS. $G_{\mathrm{r}_{j n}, \mathrm{~d}_{u n}}^{k}=\left|h_{\mathrm{r}_{j n}, \mathrm{~d}_{u n}}^{k}\right|^{2}$ denotes the channel gain of subcarrier $k$ from $\mathrm{r}_{j n}$ to $\mathrm{d}_{u n} \cdot G_{\mathrm{s}_{n^{\prime}}, \mathrm{d}_{u n}}^{k}=\left|h_{\mathrm{s}_{n^{\prime}}, \mathrm{d}_{u n}}^{k}\right|^{2}$ denotes the channel gain of subcarrier $k$ from $s_{n^{\prime}}$ to $d_{u n}$.

The maximum achievable rate for the subcarrier $k$ when allocated to $\mathrm{d}_{u n}$ is given by [3]

$$
R_{u j n, 2}^{k}=\min \left(\ln \left(1+\Gamma_{\mathrm{r}_{j n}}^{k}\right), \ln \left(1+\Gamma_{\mathrm{d}_{u n}, t_{2}}^{k}\right)\right)
$$

in nats/two-TSs.

Let us describe further the direct data transmission in cell $n$. During the first TS, $\mathrm{s}_{n}$ broadcasts $\sqrt{P_{\mathrm{s}_{n}, t_{1}}^{k}} x_{\mathrm{s}_{n}, t_{1}}^{k}$ at subcarrier $k$. The targeted MS $\mathrm{d}_{u n}$ receives signals from all BSs. Finally, the signal received by $\mathrm{d}_{u n}$ at subcarrier $k$ can be expressed as

$$
\begin{aligned}
z_{\mathrm{d}_{u n}, t_{1}}^{k}= & \sqrt{P_{\mathrm{s}_{n}, t_{1}}^{k}} h_{\mathrm{s}_{n}, \mathrm{~d}_{u n}}^{k} x_{\mathrm{s}_{n}, t_{1}}^{k}+v_{\mathrm{d}_{u n}, t_{1}}^{k} \\
& +\sum_{n^{\prime}=1, n^{\prime} \neq n}^{N} \sqrt{P_{\mathrm{s}_{n^{\prime}}, t_{1}}^{k}} h_{\mathrm{s}_{n^{\prime}}, \mathrm{d}_{u n}}^{k} x_{\mathrm{s}_{n^{\prime}}, t_{1}}^{k}
\end{aligned}
$$

where $v_{\mathrm{d}_{u n}, t_{1}}^{k}$ denotes the AWGN corrupting $\mathrm{d}_{u n}$ at subcarrier $k$ during the first TS.

During the second time slot, another symbol $\sqrt{P_{\mathrm{s}_{n}, t_{2}}^{k}} x_{\mathrm{s}_{n}, t_{2}}^{k}$ is broadcast by $\mathrm{s}_{n}$ at subcarrier $k$ and received 
by the same MS $d_{u n}$. The received signal can be expressed as:

$$
\begin{aligned}
z_{\mathrm{d}_{u n}, t_{2}}^{k}= & \sqrt{P_{\mathrm{s}_{n}, t_{2}}^{k}} h_{\mathrm{s}_{n}, \mathrm{~d}_{u n}}^{k} x_{\mathrm{s}_{n}, t_{2}}^{k}+v_{\mathrm{d}_{u n}, t_{2}}^{k} \\
& +\sum_{n^{\prime}=1, n^{\prime} \neq n}^{N} \sqrt{P_{\mathrm{s}_{n^{\prime}}, t_{2}}^{k}} h_{\mathrm{s}_{n^{\prime}}, \mathrm{d}_{u n}}^{k} x_{\mathrm{s}_{n^{\prime}}, t_{2}}^{k} \\
& +\sum_{n^{\prime}=1, n^{\prime} \neq n}^{N} \sum_{j^{\prime}=1}^{J} \sqrt{P_{\mathrm{r}_{j^{\prime} n^{\prime}}}^{k}} h_{\mathrm{r}_{j^{\prime} n^{\prime}}, \mathrm{d}_{u n}}^{k} x_{\mathrm{r}_{j^{\prime} n^{\prime}}}^{k} .
\end{aligned}
$$

Thus, the achievable sum rate for subcarrier $k$ during two TSs is given by

$$
R_{u n, 1}^{k}=\ln \left(1+\Upsilon_{\mathrm{d}_{u n}, t_{1}}^{k}\right)+\ln \left(1+\Upsilon_{\mathrm{d}_{u n}, t_{2}}^{k}\right)
$$

in nats/two-TSs, where

$$
\Upsilon_{\mathrm{d}_{u n}, t_{1}}^{k}=\frac{P_{\mathrm{s}_{n}, t_{1}}^{k} G_{\mathrm{s}_{n}, \mathrm{~d}_{u n}}^{k}}{f_{\mathrm{d}_{u n}, t_{1}}^{k}}
$$

denotes the SINR associated with the decoding of $x_{\mathrm{s}_{n}, t_{1}}^{k}$ from $y_{\mathrm{d}_{u n}, t_{1}}^{k}$ at $\mathrm{d}_{u n}$ during the first TS. $f_{\mathrm{d}_{u n}, t_{1}}^{k}=\sigma^{2}+$ $\sum_{n^{\prime}=1, n^{\prime} \neq n}^{N} P_{\mathrm{s}_{n^{\prime}}, t_{1}}^{k} G_{\mathrm{s}_{n^{\prime}}, \mathrm{d}_{u n}}^{k}$ denotes the sum power of the AWGN and the interference received by $\mathrm{d}_{u n}$ at subcarrier $k$ during the first TS.

$$
\Upsilon_{\mathrm{d}_{u n}, t_{2}}^{k}=\frac{P_{\mathrm{s}_{n}, t_{2}}^{k} G_{\mathrm{s}_{n}, \mathrm{~d}_{u n}}^{k}}{f_{\mathrm{d}_{u n}, t_{2}}^{k}}
$$

denotes the SINR associated with the decoding of $x_{\mathrm{s}_{n}, t_{2}}^{k}$ from $y_{\mathrm{d}_{u n}, t_{2}}^{k}$ at $\mathrm{d}_{u n}$ during the second TS.

\subsection{RA problem formulation}

In order to formulate the RA problem, we now introduce binary variables $a_{u n}^{k}$ and $b_{u j n}^{k}$ to describe the mode selection and subcarrier assignment in both TSs. To be more specific, $a_{u n}^{k}=1$ indicates that subcarrier $k$ is allocated for data transmission to $\mathrm{d}_{u n}$ in direct mode during two TSs. $b_{u j n}^{k}=1$ indicates that subcarrier $k$ is allocated for data transmission to $\mathrm{d}_{u n}$ aided by $\mathrm{r}_{j n}$.

We consider maximizing the sum rate of all MSs in all cells under per cell total power constraints. The optimization variables are the transmission mode for each subcarrier, the subcarrier assignments and the power allocations at the BSs and the RSs. According to the system model, the considered RA problem can be formulated as follows:

$$
\begin{aligned}
& \max _{\mathbf{P}, \mathbf{A}, \mathbf{B}} R(\mathbf{P}, \mathbf{A}, \mathbf{B}) \\
& \text { s.t. } \\
& \text { C1: }\left(\sum_{u=1}^{U} a_{u n}^{k}+\sum_{u=1}^{U} \sum_{j=1}^{J} b_{u j n}^{k}\right) \leq 1, \forall n, k, \\
& \text { C2: } a_{u n}^{k} \in\{0,1\}, b_{u j n}^{k} \in\{0,1\}, \forall u, j, n, k, \\
& \text { C3: } \sum_{k=1}^{K} P_{\mathrm{s}_{n}, t_{1}}^{k}+\sum_{k=1}^{K} P_{\mathrm{s}_{n}, t_{2}}^{k}+\sum_{j=1}^{J} \sum_{k=1}^{K} P_{\mathrm{r}_{j n}}^{k} \leq P_{t, n}, \forall n, \\
& \text { C4: } P_{\mathrm{s}_{n}, t_{1}}^{k} \geq 0, P_{\mathrm{s}_{n}, t_{2}}^{k} \geq 0, P_{\mathrm{r}_{j n}}^{k} \geq 0, \forall j, n, k, \\
& \text { C5 : } P_{\mathrm{s}_{n}, t_{1}}^{k}\left(1-\left(\sum_{u=1}^{U} a_{u n}^{k}+\sum_{u=1}^{U} \sum_{j=1}^{J} b_{u j n}^{k}\right)\right)=0, \forall n, k \\
& \text { C6 : } P_{\mathrm{s}_{n}, t_{2}}^{k}\left(1-\sum_{u=1}^{U} a_{u n}^{k}\right)=0, \forall n, k, \\
& \text { C7 : } P_{\mathrm{r}_{j_{n}}}^{k}\left(1-\sum_{u=1}^{U} b_{u j n}^{k}\right)=0, \forall j, n, k
\end{aligned}
$$

where

$$
\begin{aligned}
R(\mathbf{P}, \mathbf{A}, \mathbf{B})= & \sum_{n=1}^{N} \sum_{u=1}^{U} \sum_{k=1}^{K} a_{u n}^{k} R_{u n, 1}^{k}\left(\mathbf{P}_{\mathbf{n}}^{\mathbf{k}}\right) \\
& +\sum_{n=1}^{N} \sum_{u=1}^{U} \sum_{j=1}^{J} \sum_{k=1}^{K} b_{u j n}^{k} R_{u j n, 2}^{k}\left(\mathbf{P}_{\mathbf{n}}^{\mathbf{k}}\right),
\end{aligned}
$$

$\mathbf{P}=\left[\left[\mathbf{P}_{1}\right]^{\mathrm{T}}, \ldots,\left[\mathbf{P}_{N}\right]^{\mathrm{T}}\right]^{\mathrm{T}}, \mathbf{P}_{n}=\left[\left[\mathbf{P}_{\mathbf{n}}^{\mathbf{1}}\right]^{\mathrm{T}}, \ldots,\left[\mathbf{P}_{\mathbf{n}}^{\mathrm{K}}\right]^{\mathrm{T}}\right]^{\mathrm{T}}$, $\mathbf{P}_{\mathbf{n}}^{\mathbf{k}}=\left[P_{\mathrm{s}_{n}, t_{1}}^{k}, P_{\mathrm{s}_{n}, t_{2}}^{k}, P_{\mathrm{r}_{1 n}}^{k}, \ldots, P_{\mathrm{r}_{1 n}}^{k}\right]^{\mathrm{T}}, \mathbf{A}=\left\{a_{u n}^{k}\right\}, \mathbf{B}=$ $\left\{b_{u j n}^{k}\right\}, P_{t, n}$ denotes the available total power in cell $n$.

Here, $C 1$ and $C 2$ ensure that each subcarrier $k$ during the first TS can select only one mode (direct/relay-aided) to transmit data towards only one MS $\mathrm{d}_{u n}$. If the relayaided mode is selected, the data transmission can be helped by only one RS $\mathrm{r}_{j n}$. Moreover, $C 3$ and $C 4$ ensure that the consumed sum power for each cell is less than its available sum power. This type of power constraints 
gives an upper bound of the system performance. In practice, each node (BS, RS) in each cell will have an individual power constraint. Finally, $C 5, C 6$, and $C 7$ guarantee that no data is transmitted on an unused subcarrier and subcarrier $k$ is used by only one node (either the BS or the RS) in each cell during the second TS.

\section{SCLD-based coordinate ascent RA algorithm}

\subsection{The overall RA algorithm}

In order to solve problem (11), an iterative coordinate ascent approach is adopted. Each iteration is carried out in two stages: the MSSA stage and the PA stage. We introduce integer $m$ to indicate the iteration number and add superscript $m$ to variables obtained at the end of iteration $m$. We now propose the overall RA algorithm, as depicted in Algorithm 1.

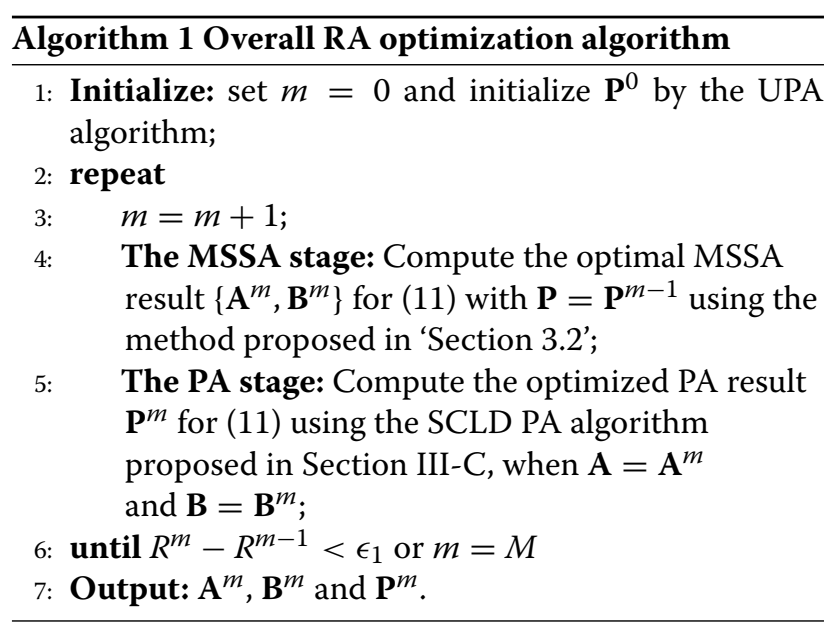

Specifically in the proposed RA algorithm, we first set $m=0$ and initialize the power $\mathbf{P}^{0}$ either by the uniform power allocation (UPA) or by using the optimal algorithm, as proposed in [16] when CCI is ignored. Each iteration consists of the MSSA stage and the PA stage. During the MSSA stage of iteration $m$, we set $\mathbf{P}=\mathbf{P}^{m-1}$ and decouple problem (11) into $n * k$ integer linear programs (ILPs), which can easily be solved in linear time, as will be shown in 'Section 3.2'. After optimization, $\left\{\mathbf{A}^{m}, \mathbf{B}^{m}\right\}$ will be obtained. Finally, we have $R\left(\mathbf{P}^{m-1}, \mathbf{A}^{m-1}, \mathbf{B}^{m-1}\right) \leq$ $R\left(\mathbf{P}^{m-1}, \mathbf{A}^{m}, \mathbf{B}^{m}\right)$.

During the PA stage of iteration $m$, we set $\mathbf{A}=\mathbf{A}^{m}$, $\mathbf{B}=\mathbf{B}^{m}$ and solve problem (11) using the SCLD PA algorithm proposed in 'Section 3.3'. The output delivered is denoted as $\mathbf{P}^{m}$. As will be shown in 'Section 3.3', the SCLD PA algorithm approximates the original non-convex problem into a series of convex problems. The solutions of the approximated convex problems converge to a local optimum satisfying the Karush-Kuhn-Tucker (KKT) conditions of the non-convex problem. After convergence, the power vector $\mathbf{P}^{m}$ will be the output. Finally, we have $R\left(\mathbf{P}^{m-1}, \mathbf{A}^{m}, \mathbf{B}^{m}\right) \leq R\left(\mathbf{P}^{m}, \mathbf{A}^{m}, \mathbf{B}^{m}\right)$.

Considering the proposed RA algorithm, we now have the following:

$$
\begin{aligned}
& R\left(\mathbf{P}^{m-1}, \mathbf{A}^{m-1}, \mathbf{B}^{m-1}\right) \\
E 1: \leq & R\left(\mathbf{P}^{m-1}, \mathbf{A}^{m}, \mathbf{B}^{m}\right) \\
E 2: \leq & R\left(\mathbf{P}^{m}, \mathbf{A}^{m}, \mathbf{B}^{m}\right)
\end{aligned}
$$

where $E 1$ and $E 2$ are due to the MSSA stage and the PA stage, respectively. This means that Algorithm 1 yields non-decreasing sum rates along with iterations. Moreover, due to the total power constraint in each cell, the optimum sum rate is upper bounded and the sum rate values will not increase indefinitely along with iterations. This means that the iterations will eventually converge [31]. Algorithm 1 will stop when the sum rate increase is below a prescribed value $\epsilon_{1}$ or when $m$ reaches a prescribed value $M$.

At the end of iteration $m$, a local optimum of the formulated problem is obtained at the PA stage, which is then improved at the MSSA stage of the next iteration. After that, a better local optimum can be calculated at the PA stage of iteration $m+1$. Finally, a good local optimum can be obtained after convergence. Note that each stage is carried out with analytical equations. Thus, the proposed RA algorithm has a lower-complexity compared to the centralized algorithm (CA) of [25]. As will be elaborated in 'Section 3.2' and 'Section 3.3', the proposed MSSA algorithm can be implemented distributively in each cell and the SCLD PA algorithm can be implemented semidistributively in each cell. Thus, the proposed iterative RA algorithm can be implemented semi-distributively in each cell with the help of a central controller (CC) exchanging messages among cells.

\subsection{MSSA optimization}

In this subsection, the MSSA stage for iteration $m$ is considered. After setting $\mathbf{P}$ to $\mathbf{P}^{m}$, problem (11) can be rewritten as follows:

$$
\begin{aligned}
& \max _{\mathbf{A}, \mathbf{B}} R\left(\mathbf{P}^{m}, \mathbf{A}, \mathbf{B}\right) \\
& \text { s.t. } C 1, C 2, C 5, C 6, C 7 .
\end{aligned}
$$

Note that each subcarrier in each cell has independent constraints. Therefore, problem (13) can be decoupled into $N * K$ subproblems. Specifically, subproblem $i$, which 
corresponds to subcarrier $k_{0}$ in cell $n_{0}$ is formulated as the following:

$$
\begin{aligned}
& \max _{\mathbf{A}_{i}, \mathbf{B}_{i}} R_{i}\left(\mathbf{P}^{m}, \mathbf{A}_{i}, \mathbf{B}_{i}\right) \\
& \text { s.t. } \\
& C 1^{\prime}: \sum_{u} a_{u n_{0}}^{k_{0}}+\sum_{u} \sum_{j} b_{u j n_{0}}^{k_{0}} \leq 1, \\
& C 2^{\prime}: a_{u n_{0}}^{k_{0}} \in\{0,1\}, b_{u j n_{0}}^{k_{0}} \in\{0,1\}, \forall u, j, \\
& C 5^{\prime}: P_{\mathrm{s}_{n_{0}}, t_{1}}^{k_{0}, m-1}\left(1-\left(\sum_{u=1}^{U} a_{u n_{0}}^{k_{0}}+\sum_{u=1}^{U} \sum_{j=1}^{J} b_{u j n_{0}}^{k_{0}}\right)\right)=0, \\
& C 6^{\prime}: P_{\mathrm{s}_{n_{0}}, t_{2}}^{k_{k_{0}, m-1}}\left(1-\sum_{u=1}^{U} a_{u n_{0}}^{k_{0}}\right)=0, \\
& C 7^{\prime}: P_{\mathrm{r}_{j n_{0}}}^{k_{0}, m-1}\left(1-\sum_{u=1}^{U} b_{u j n_{0}}^{k_{0}}\right)=0, \forall j,
\end{aligned}
$$

where $\mathbf{A}_{i}=\left\{a_{u n}^{k} \mid n=n_{0}, k=k_{0}\right\}, \mathbf{B}_{i}=\left\{b_{\text {ujn }}^{k} \mid n=n_{0}, k=k_{0}\right\}$ and $R_{i}$ are given by

$$
R_{i}=\sum_{u} a_{u n_{0}}^{k_{0}} R_{u n_{0}, 1}^{k_{0}}\left(\mathbf{P}^{m}\right)+\sum_{u} \sum_{j} b_{u j n_{0}}^{k_{0}} R_{u j n_{0}, 2}^{k_{0}}\left(\mathbf{P}^{m}\right) .
$$

As will be explained later, problem (14) has the same optimum solution as the following problem, where the constraints $C 5^{\prime}-C 7^{\prime}$ are removed.

$$
\begin{aligned}
& \max _{\mathbf{A}_{i}, \mathbf{B}_{i}} R_{i}\left(\mathbf{P}^{m-1}, \mathbf{A}_{i}, \mathbf{B}_{i}\right) \\
& \text { s.t. } C 1^{\prime}, C 2^{\prime} .
\end{aligned}
$$

Specifically, $\forall n_{0}, k_{0}$, when $P_{\mathrm{s}_{n_{0}}, t_{1}}^{k_{0}, m-1}>0$, we certainly have $R_{u n_{0}, 1}^{k_{0}}>0, \forall u$. Therefore at the optimum of problem (15), we have $\sum_{u=1}^{U} a_{u n_{0}}^{k_{0}}+\sum_{u=1}^{U} \sum_{j=1}^{J} b_{u j n_{0}}^{k_{0}}=1$, meaning that this subcarrier $k_{0}$ should be used for data transmission. Otherwise, we can still improve $R_{i}$ by randomly selecting one variable of the set $\left\{a_{u n}^{k} \mid k=k_{0}, n=n_{0}\right\}$ and setting it to 1 . Thus in this case, we have $P_{\mathrm{s}_{n_{0}}, t_{1}}^{k_{0}, m-1}\left(1-\left(\sum_{u=1}^{U} a_{u n_{0}}^{k_{0}}+\sum_{u=1}^{U} \sum_{j=1}^{J} b_{u j n_{0}}^{k_{0}}\right)\right)=0$. Also, when $P_{\mathrm{s}_{n_{0}}, t_{1}}^{k_{0}, m-1}=0$, we certainly have $P_{\mathrm{s}_{n_{0}}, t_{1}}^{k_{0}, m-1}\left(1-\left(\sum_{u=1}^{U} a_{u n_{0}}^{k_{0}}+\sum_{u=1}^{U} \sum_{j=1}^{J} b_{u j n_{0}}^{k_{0}}\right)\right)=0$. Thus at the optimum of problem (15), the constraint $C 6.5^{\prime}$ is fulfilled. Similarly, at the optimum of problem (15), the constraints $C 6.6^{\prime}$ and $C 6.7^{\prime}$ are fulfilled. This means that the optimum solution of problem (15) is also feasible for problem (14). Note that problem (14) is the same as problem (15) except that its feasible set is a subset of that of problem (15). Thus, the optimum solution of problem (15) is also the optimum solution of problem (14).
According to the expression of $R_{i}$ and the constraints $\left(C 1^{\prime}, C 2^{\prime}\right)$, this subproblem (15) is further denoted as

$$
\begin{aligned}
& \max _{\mathbf{A}_{i}, \mathbf{B}_{i}} \sum_{u=1}^{U} a_{u n_{0}}^{k_{0}} R_{u n_{0}, 1}^{k_{0}}\left(\mathbf{P}^{m-1}\right)+\sum_{u=1}^{U} \sum_{j=1}^{J} b_{u j n_{0}}^{k_{0}} R_{u j n_{0}, 2}^{k_{0}}\left(\mathbf{P}^{m-1}\right), \\
& C 1^{\prime}: \sum_{u} a_{u n_{0}}^{k_{0}}+\sum_{u} \sum_{j} b_{u j n_{0}}^{k_{0}} \leq 1, \\
& C 2^{\prime}: a_{u n_{0}}^{k_{0}} \in\{0,1\}, b_{u j n_{0}}^{k_{0}} \in\{0,1\}, \forall u, j .
\end{aligned}
$$

It is obvious that (15) can easily be solved by searching the optimal mode, MS, and RS which provide the maximum rate for subcarrier $k_{0}$ in cell $n_{0}$ with $\mathbf{P}=\mathbf{P}^{m-1}$.

\subsection{PA optimization}

In this subsection, the PA stage for iteration $m$ is considered. After setting the indicators to $\left\{\mathbf{A}^{m}, \mathbf{B}^{m}\right\}$, the transmission modes are fixed in all cells. We denote by $\mathcal{S}_{\text {un }}(d)$ the set of subcarriers allocated to MS $d_{u n}$ in direct mode and $\mathcal{S}_{u j n}(r)$ the set of subcarriers allocated to MS $d_{u n}$ and $\mathrm{RS} r_{j n}$ in relay-aided mode. Then, the objective function of problem (11) can be rewritten as $R\left(\mathbf{P}, \mathbf{A}^{m}, \mathbf{B}^{m}\right)$, given by the following:

$$
\begin{gathered}
\sum_{n}\left(\sum_{k \in \mathcal{S}_{u n}(d)} \log \left(1+\Upsilon_{\mathrm{d}_{u n}, t_{1}}^{k}\right)+\log \left(1+\Upsilon_{\mathrm{d}_{u n}, t_{2}}^{k}\right)\right. \\
\left.\quad+\sum_{k \in \mathcal{S}_{u j n}(r)} \log \min \left(1+\Gamma_{\mathrm{r}_{j n}}^{k}, 1+\Gamma_{\mathrm{d}_{u n, t_{2}}}^{k}\right)\right) .
\end{gathered}
$$

Note that $R$ is a non-convex function due to the presence of interfering power terms in the denominators of $\Upsilon_{\mathrm{d}_{u n}, t_{1}}^{k}, \Upsilon_{\mathrm{d}_{u n}, t_{2}}^{k}, \Gamma_{\mathrm{r}_{j n}}^{k}$, and $\Gamma_{\mathrm{d}_{u n, t_{2}}}^{k}$. To solve problem (11), we first replace it with a minimization problem that is then solved by using the SCLD PA algorithm.

The equivalent minimization problem is obtained in two steps. Problem (11) is first converted into an equivalent one given by

$$
\begin{aligned}
& \min _{\mathbf{P}}-R\left(\mathbf{P}, \mathbf{A}^{m}, \mathbf{B}^{m}\right) \\
& \text { s.t. } C 3-C 7,
\end{aligned}
$$

where $-R\left(\mathbf{P}, \mathbf{A}^{m}, \mathbf{B}^{m}\right)$ is given by

$$
\begin{gathered}
\sum_{n}\left(\sum_{k \in \mathcal{S}_{u n}(d)}\left(\log \frac{1}{1+\Upsilon_{\mathrm{d}_{u n}, t_{1}}^{k}}+\log \frac{1}{1+\Upsilon_{\mathrm{d}_{u n}, t_{2}}^{k}}\right)\right. \\
\left.\sum_{k \in \mathcal{S}_{u j n}(r)} \log \max \left(\frac{1}{1+\Gamma_{\mathrm{r}_{j n}}^{k}}, \frac{1}{1+\Gamma_{\mathrm{d}_{u n, t_{2}}}^{k}}\right)\right)
\end{gathered}
$$




$$
\begin{aligned}
& \frac{1}{1+\Upsilon_{\mathrm{d}_{u n}, t_{1}}^{k}}=\frac{f_{\mathrm{d}_{u n}, t_{1}}^{k}}{f_{\mathrm{d}_{u n}, t_{1}}^{k}+P_{\mathrm{s}_{n}, t_{1}}^{k} G_{\mathrm{s}_{n}, \mathrm{~d}_{u n}}^{k}}, \\
& \frac{1}{1+\Upsilon_{\mathrm{d}_{u n}, t_{2}}^{k}}=\frac{f_{\mathrm{d}_{u n}, t_{2}}^{k}}{f_{\mathrm{d}_{u n}, t_{2}}^{k}+P_{\mathrm{s}_{n}, t_{2}}^{k} G_{\mathrm{s}_{n}, \mathrm{~d}_{u n}}^{k}}, \\
& \frac{1}{1+\Gamma_{\mathrm{d}_{u n}, t_{2}}^{k}}=\frac{f_{\mathrm{d}_{u n}, t_{2}}^{k}}{f_{\mathrm{d}_{u n}, t_{2}}^{k}+P_{\mathrm{r}_{j n}}^{k} G_{\mathrm{r}_{j n}, \mathrm{~d}_{u n}}^{k}}, \\
& \frac{1}{1+\Gamma_{\mathrm{r}_{j n}}^{k}}=\frac{f_{\mathrm{r}_{j n}}^{k}}{f_{\mathrm{r}_{j n}}^{k}+P_{\mathrm{s}_{n}, t_{1}}^{k} G_{\mathrm{s}_{n}, \mathrm{r}_{j n}}^{k}} .
\end{aligned}
$$

After that, another equivalent formulation is obtained by introducing slack variables $\Psi_{r}=\left\{\Psi_{r}^{n k}, \forall n, k \in\right.$ $\left.\mathcal{S}_{\text {ujn }}(r)\right\}$. Then, problem (16) can be formulated as follows:

$$
\begin{aligned}
& \min _{\mathbf{P}, \Psi_{r}} \sum_{n}\left(\sum_{k \in \mathcal{S}_{u n}(d)}\left(\log \frac{f_{\mathrm{d}_{u n}, t_{1}}^{k}}{g_{\mathrm{d}_{u n}, t_{1}}^{k}} \frac{f_{\mathrm{d}_{u n}, t_{2}}^{k}}{g_{\mathrm{s}_{n} \mathrm{~d}_{u n}, t_{2}}^{k}}+\sum_{k \in \mathcal{S}_{u j n}(r)} \Psi_{r}^{n k}\right)\right. \\
& \text { s.t. } C 3-C 7 \\
& \text { C8: } \log \frac{f_{\mathrm{r}_{j n}}^{k}}{g_{\mathrm{r}_{j n}}^{k}}-\Psi_{r}^{n k} \leq 0, \forall n, k \in \mathcal{S}_{u j n}(r), \\
& \text { C9: } \log \frac{{\frac{\mathrm{d}_{u n}, t_{2}}{k}}_{g_{\mathrm{r}_{j n} \mathrm{~d}_{u n}, t_{2}}^{k}}^{k}}{\Psi_{r}} \leq \Psi_{r}^{n k} \leq 0, \forall n, k \in \mathcal{S}_{u j n}(r),
\end{aligned}
$$

where $g_{\mathrm{d}_{u n}, t_{1}}^{k}=f_{\mathrm{d}_{u n}, t_{1}}^{k}+P_{\mathrm{s}_{n}, t_{1}}^{k} G_{\mathrm{s}_{n}, \mathrm{~d}_{u n}}^{k}, g_{\mathrm{s}_{n} \mathrm{~d}_{u n}, t_{2}}^{k}=f_{\mathrm{d}_{u n}, t_{2}}^{k}+$ $P_{\mathrm{s}_{n}, t_{2}}^{k} G_{\mathrm{s}_{n}, \mathrm{~d}_{u n}}^{k}, g_{\mathrm{r}_{j n} \mathrm{~d}_{u n}, t_{2}}^{k}=f_{\mathrm{d}_{u n}, t_{2}}^{k}+P_{\mathrm{r}_{j n}}^{k} G_{\mathrm{r}_{j n}, \mathrm{~d}_{u n}}^{k}$, and $g_{\mathrm{r}_{j n}}^{k}=f_{\mathrm{r}_{j n}}^{k}+$ $P_{\mathrm{s}_{n}, t_{1}}^{k} G_{\mathrm{s}_{n}, \mathrm{r}_{j n}}^{k}$.

Problem (22) is still non-convex. In order to solve it, we now propose the SCLD PA algorithm. To derive it, two steps are involved. During the first step, a convex approximation of problem (22) is constructed. We first use the method of Lemma 1 in [33] to condense all denominator posynomials $\left\{g_{\mathrm{d}_{u n}, t_{1}}^{k}, g_{s_{n} \mathrm{~d}_{u n}, t_{2}}^{k}, g_{\mathrm{r}_{j n} \mathrm{~d}_{u n}, t_{2}}^{k}, g_{\mathrm{r}_{j n}}^{k}\right\}$ into monomials $\left\{\hat{g}_{\mathrm{d}_{u n}, t_{1}}^{k}, \hat{g}_{\mathrm{s}_{n} \mathrm{~d}_{u n}, t_{2}}^{k}, \hat{g}_{\mathrm{r}_{j n} \mathrm{~d}_{u n}, t_{2}}^{k}, \hat{g}_{\mathrm{r}_{j n}}^{k}\right\}$ using tentative PA results $\mathbf{P}_{0}=\left\{P_{\mathrm{s}_{n}, t_{1}, 0}^{k}, P_{\mathrm{s}_{n}, t_{2}, 0}^{k}, P_{\mathrm{r}_{j n}, 0}^{k}\right\}$. Functions $h_{1}^{n k}=\log \frac{f_{\mathrm{r}_{j n}}^{k}}{g_{r_{j n}}^{k}}-\Psi_{r}^{n k}, h_{2}^{n k}=\log \frac{f_{\mathrm{d}_{u n}, t_{2}}^{k}}{g_{\mathrm{r}_{j n} \mathrm{~d}_{u n}, t_{2}}^{k}}-\Psi_{r}^{n k}$, and $h_{3}^{n k}=$ $\sum_{n}\left(\sum_{k \in \mathcal{S}_{u n}(d)} \log \frac{f_{\mathrm{d}_{u n}, t_{1}}^{k}}{g_{\mathrm{d}_{u n}, t_{1}}^{k}} \frac{f_{\mathrm{d}_{u n}, t_{2}}^{k}}{g_{\mathrm{s}_{n}}^{k} \mathrm{~d}_{u n}, t_{2}}+\sum_{k \in \mathcal{S}_{u j n}(r)} \Psi_{r}^{n k}\right)$ are approximated by $\hat{h}_{1}^{n k}=\log \frac{f_{r_{j n}}^{k}}{\hat{g}_{r_{j n}}^{k}}-\Psi_{r}^{n k}, \hat{h}_{2}^{n k}=\log \frac{f_{\mathrm{d}_{u n}, t_{2}}^{k}}{\hat{g}_{\mathrm{r}_{j n} \mathrm{~d} u n, t_{2}}}-\Psi_{r}^{n k}$, and $\hat{h}_{3}^{n k}=\sum_{n}\left(\sum_{k \in \mathcal{S}_{u n}(d)} \log \frac{f_{\mathrm{d}_{u n}, t_{1}}^{k}}{\hat{g}_{\mathrm{d}_{u n}, t_{1}}^{k}} \frac{f_{\mathrm{d}_{u n}, t_{2}}^{k}}{\hat{g}_{\mathrm{s}_{n} \mathrm{~d}_{u n}, t_{2}}^{k}}+\sum_{k \in \mathcal{S}_{u j n}(r)} \Psi_{r}^{n k}\right)$, respectively. Then, by introducing the logarithmic change of the variables (e.g., $\tilde{x}=\log x$ ), the approximated problem is formulated as the following:

$$
\begin{aligned}
& \underset{\tilde{\mathbf{P}}, \tilde{\Psi}_{r}}{\operatorname{minimize}} \sum_{n} R^{n}\left(\tilde{\mathbf{P}}, \tilde{\Psi}_{r}\right) \\
& \text { s.t. } C 3, C 5-C 7 \text {, } \\
& C 4: \quad \frac{1}{P_{T}^{n}} \sum_{k}\left(e^{\tilde{P}_{\mathrm{r}_{n n}}^{k}}+e^{\tilde{P}_{\mathrm{s}_{n}, t_{1}}^{k}}+e^{\tilde{P}_{\mathrm{s}_{n}, t_{2}}^{k}}\right) \leq 1, \forall n, \\
& \text { C8 : } \quad \log \frac{f_{\mathrm{r}_{j n}}^{k}\left(e^{\tilde{\mathbf{P}}}\right)}{\hat{g}_{\mathbf{r}_{j n}}^{k}\left(e^{\tilde{\mathbf{P}}}\right) e^{\tilde{\Psi}_{r}^{n k}}} \leq 0, \forall n, k \in \mathcal{S}_{u j n}(r), \\
& \text { C9 : } \quad \log \frac{f_{\mathrm{d}_{u n}, t_{2}}^{k}\left(e^{\tilde{\mathbf{P}}}\right)}{\hat{g}_{\mathrm{r}_{j n} \mathrm{~d}_{u n}, t_{2}}^{k}\left(e^{\tilde{\mathbf{P}}}\right) e^{\tilde{\Psi}_{r}^{n k}}} \leq 0, \forall n, k \in \mathcal{S}_{u j n}(r),
\end{aligned}
$$

where $R^{n}\left(\tilde{\mathbf{P}}, \tilde{\Psi}_{r}\right)$ is given by

$$
\sum_{k \in \mathcal{S}_{u n}(d)} \log \frac{f_{\mathrm{d}_{u n}, t_{1}}^{k}\left(e^{\tilde{\mathbf{p}}}\right)}{\hat{g}_{\mathrm{d}_{u n}, t_{1}}^{k}\left(e^{\tilde{\mathbf{P}}}\right)} \frac{f_{\mathrm{d}_{u n}, t_{2}}^{k}\left(e^{\tilde{\mathbf{p}}}\right)}{\hat{g}_{\mathrm{s}_{n} \mathrm{~d}_{u n}, t_{2}}^{k}\left(e^{\tilde{\mathbf{P}}}\right)}+\sum_{k \in \mathcal{S}_{u j n}(r)} \log e^{\tilde{\Psi}_{r}^{n k}}
$$

$\tilde{\mathbf{P}}$ denotes the vector of logarithmic power variables, and $\tilde{\Psi}_{r}$ denotes the vector of logarithmic slack variables $\tilde{\Psi}_{r}^{n k}$.

Note that the method we used in this step belongs to the class of successive convex approximation methods [34]. After some mathematical calculations, it is easy to verify that all the approximations of problem (22) satisfy the three conditions proposed in [34] for the convergence of the successive approximation method. By denoting $\Theta=$ $\left\{\mathbf{P}, \Psi_{r}\right\}$, the three conditions are listed as follows:

1. Bounding condition: $\forall \Theta$,

$$
h_{i}^{n, k}(\Theta) \leq \tilde{h}_{i}^{n, k}(\Theta), \forall i=1,2,3 .
$$

2. Tightness condition: At the beginning of iteration $t$,

$$
h_{i}^{n, k}\left(\Theta^{m, t-1}\right)=\tilde{h}_{i}^{n, k}\left(\Theta^{m, t-1}\right), \forall i=1,2,3 .
$$

3. Differential condition: At the beginning of iteration $t$, $\forall \theta \in \Theta$,

$$
\frac{\partial h_{i}^{n, k}\left(\Theta^{m, t-1}\right)}{\partial \theta}=\frac{\partial \tilde{h}_{i}^{n, k}\left(\Theta^{m, t-1}\right)}{\partial \theta}, \forall i=1,2,3 .
$$


During the second step, the Lagrange dual method is applied to solve the approximated convex problem. Let us denote the Lagrange multipliers of $C 4, C 5$, and $C 6$ by $\lambda=\left\{\lambda_{n}\right\}, \mu=\left\{\mu_{n k}\right\}_{k \in \mathcal{S}_{u j n}(r)}$, and $v=\left\{v_{n k}\right\}_{k \in \mathcal{S}_{u j n}(r)}$, respectively. Then, the dual problem of (23) is written as $\max \left(\min L\left(\lambda, \mu, v, \tilde{\mathbf{P}}, \tilde{\Psi}_{r}\right)\right)$. Here, $L\left(\lambda, \mu, v, \tilde{\mathbf{P}}, \tilde{\Psi}_{r}\right)$ denotes $\lambda, \mu, \nu \tilde{\mathbf{P}}, \tilde{\Psi}_{r}$

the Lagrange of problem (16).

After taking the derivative of $L$ with relation to (w.r.t.) $\tilde{\Psi}_{r}^{n k}$ and setting it to 0 , we obtain $\mu_{n k}+v_{n k}=1, \forall n, k \in$ $\mathcal{S}_{u j n}(r)$. By applying it, the dual variables are updated with the subgradient method as the following:

$$
\begin{aligned}
& \lambda_{n}^{(i+1)}=\lambda_{n}^{(i)}+\delta_{\lambda, n}^{(i)}\left(\frac{1}{P_{T}^{n}} \sum_{k}\left(P_{\mathrm{r}_{j n}}^{k,(i)}+P_{\mathrm{s}_{n}}^{k,(i)}+P_{\mathrm{s}_{n}, t_{2}}^{k,(i)}\right)-1\right), \\
& \mu_{n k}^{(i+1)}=\mu_{n k}^{(i)}+\delta_{\mu, n k}^{(i)} \log \frac{f_{\mathrm{r}_{j n}}^{k}\left(\mathbf{P}^{(i)}\right) \hat{g}_{\mathrm{r}_{j n} \mathrm{~d}_{u n}, t_{2}}^{k}\left(\mathbf{P}^{(i)}\right)}{\hat{g}_{\mathrm{r}_{j n}}^{k}\left(\mathbf{P}^{(i)}\right) f_{\mathrm{d}_{u n}, t_{2}}^{k}\left(\mathbf{P}^{(i)}\right)}, \\
& v_{n k}^{(i+1)}=1-\mu_{n k}^{(i+1)} .
\end{aligned}
$$

Here, $\delta_{\lambda, n}^{(i)}=\alpha_{\lambda, n} / \sqrt{i}$ and $\delta_{\mu, n k}^{(i)}=\alpha_{\mu, n k} / \sqrt{i}$ are the step sizes chosen for $\lambda$ and $\mu$, respectively. We assume that each receiver in a cell $n$ can estimate the received power from each of the interfering stations (either the BS or a RS of an interfering cell). Thus, $f_{\mathrm{r}_{j n}}^{k}\left(\mathbf{P}^{(i)}\right), f_{\mathrm{d}_{u n}, t_{2}}^{k}\left(\mathbf{P}^{(i)}\right), \hat{g}_{\mathrm{r}_{j n}}^{k} \mathrm{~d}_{u n}, t_{2}\left(\mathbf{P}^{(i)}\right)$, and $\hat{g}_{\mathrm{r}_{j n}}^{k}\left(\mathbf{P}^{(i)}\right)$ can be calculated locally in the cell $n$.

We further take the derivative of $L$ w.r.t. $\tilde{P}_{\mathrm{s}_{n}, t_{1}}^{k}, \tilde{P}_{\mathrm{r}_{j n}}^{k}$, and $\tilde{P}_{S_{n}, t_{2}}^{k}$, and force them to 0 . With the logarithmic power variables transformed back to the original $P$ space, fixed-point equations for $P_{\mathrm{s}_{n}, t_{1}}^{k,(i)}, \forall n, k, P_{\mathrm{r}_{j n}}^{k,(i)}, \forall n, k \in$ $\mathcal{S}_{u j n}(r)$, and $P_{\mathrm{S}_{n}, t_{2}}^{k,(i)}, \forall n, k \in \mathcal{S}_{u n}(d)$ can be formulated as

$$
h_{4}^{n k}\left(\lambda_{n}^{(i)}, \mu^{(i)}, \mathbf{P}^{(i)}\right)=\frac{X_{1}^{n k}\left(\mathbf{P}_{0}\right)+X_{2}^{n k}\left(\mu^{(i)}, \mathbf{P}_{0}\right)}{\frac{\lambda_{n}^{(i)}}{P_{T}^{n}}+Y_{1}^{n k}\left(\mathbf{P}^{(i)}\right)+Y_{2}^{n k}\left(\mu^{(i)}, \mathbf{P}^{(i)}\right)},
$$

$h_{5}^{n k}\left(\lambda_{n}^{(i)}, v^{(i)}, \mathbf{P}^{(i)}\right)=\frac{X_{3}^{n k}\left(\mathbf{P}_{0}\right)+X_{4}^{n k}\left(v^{(i)}, \mathbf{P}_{0}\right)}{\frac{\lambda_{n}^{(i)}}{P_{T}^{n}}+Y_{3}^{n k}\left(\mathbf{P}^{(i)}\right)+Y_{4}^{n k}\left(v^{(i)}, \mathbf{P}^{(i)}\right)}$,

and

$$
h_{6}^{n k}\left(\lambda_{n}^{(i)}, v^{(i)}, \mathbf{P}^{(i)}\right)=\frac{X_{5}^{n k}\left(\mathbf{P}_{0}\right)+X_{6}^{n k}\left(v^{(i)}, \mathbf{P}_{0}\right)}{\frac{\lambda_{n}^{(i)}}{P_{T}^{n}}+Y_{5}^{n k}\left(\mathbf{P}^{(i)}\right)+Y_{6}^{n k}\left(v^{(i)}, \mathbf{P}^{(i)}\right)},
$$

respectively. Here, $\mu^{(i)}=\left\{\mu_{n k}^{(i)}\right\}, v^{(i)}=\left\{v_{n k}^{(i)}\right\}$,

$$
X_{1}^{n k}\left(\mathbf{P}_{0}\right)=\sum_{n^{\prime}, k \in \mathcal{S}_{u^{\prime} n^{\prime}}(d)} \frac{P_{\mathrm{s}_{n}, 0}^{k} G_{\mathrm{s}_{n}, \mathrm{~d}_{u^{\prime} n^{\prime}}}^{k}}{g_{\mathrm{d}_{u^{\prime} n^{\prime}, t_{1}}}^{k}\left(\mathbf{P}_{0}\right)}
$$$$
X_{2}^{n k}\left(\mu^{(i)}, \mathbf{P}_{0}\right)=\sum_{n^{\prime}, k \in \mathcal{S}_{u^{\prime} j^{\prime} n^{\prime}}(r)} \mu_{n^{\prime} k}^{(i)} \frac{P_{\mathrm{s}_{n}, 0}^{k} G_{\mathrm{s}_{n}, \mathrm{r}_{j^{\prime} n^{\prime}}}^{k}}{g_{\mathrm{r}_{j^{\prime} n^{\prime}}}^{k}\left(\mathbf{P}_{0}\right)},
$$$$
X_{3}^{n k}\left(\mathbf{P}_{0}\right)=\sum_{n^{\prime}, k \in \mathcal{S}_{u^{\prime} n^{\prime}}(d)} \frac{P_{\mathrm{r}_{j n}, 0}^{k} G_{\mathrm{r}_{j n}, \mathrm{~d}_{u^{\prime} n^{\prime}}}^{k}}{g_{\mathrm{d}_{u^{\prime} n^{\prime}}, t_{2}}^{k}\left(\mathbf{P}_{0}\right)},
$$$$
X_{4}^{n k}\left(v^{(i)}, \mathbf{P}_{0}\right)=\sum_{n^{\prime}, k \in \mathcal{S}_{u^{\prime} j^{\prime} n^{\prime}}(r)} v_{n^{\prime} k}^{(i)} \frac{P_{\mathrm{r}_{j n}, 0}^{k} G_{\mathrm{r}_{j n}, \mathrm{~d}_{u^{\prime} n^{\prime}}}^{k}}{g_{\mathrm{d}_{u^{\prime} n^{\prime}}, t_{2}}^{k}\left(\mathbf{P}_{0}\right)},
$$$$
X_{5}^{n k}\left(\mathbf{P}_{0}\right)=\sum_{n^{\prime}, k \in \mathcal{S}_{u^{\prime} n^{\prime}}(d)} \frac{P_{\mathrm{s}_{n}, 0}^{k} G_{\mathrm{s}_{n}, \mathrm{~d}_{u^{\prime} n^{\prime}}}^{k}}{g_{\mathrm{d}_{u^{\prime} n^{\prime}}, t_{2}}^{k}\left(\mathbf{P}_{0}\right)}
$$$$
X_{6}^{n k}\left(v^{(i)}, \mathbf{P}_{0}\right)=\sum_{n^{\prime}, k \in \mathcal{S}_{u^{\prime} j^{\prime} n^{\prime}}(r)} v_{n^{\prime} k}^{(i)} \frac{P_{\mathrm{s}_{n}, 0}^{k} G_{\mathrm{s}_{n}, \mathrm{~d}_{u^{\prime} n^{\prime}}}^{k}}{g_{\mathrm{d}_{u^{\prime} n^{\prime}, t_{2}}^{k}}^{k}\left(\mathbf{P}_{0}\right)},
$$$$
Y_{1}^{n k}\left(\mathbf{P}^{(i)}\right)=\sum_{n^{\prime} \neq n, k \in \mathcal{S}_{u^{\prime} n^{\prime}}(d)} \frac{G_{\mathrm{s}_{n}, \mathrm{~d}_{u^{\prime} n^{\prime}}}^{k}}{f_{\mathrm{d}_{u^{\prime} n^{\prime}}, t_{1}}^{k}\left(\mathbf{P}^{(i)}\right)},
$$

$$
\begin{aligned}
& Y_{2}^{n k}\left(\mu^{(i)}, \mathbf{P}^{(i)}\right)=\sum_{n^{\prime} \neq n, k \in \mathcal{S}_{u^{\prime} j^{\prime} n^{\prime}}(r)} \mu_{n^{\prime} k}^{(i)} \frac{G_{\mathrm{s}_{n}, \mathrm{r}^{\prime} n^{\prime}}^{k}}{f_{\mathrm{r}_{j^{\prime} n^{\prime}}}^{k}\left(\mathbf{P}^{(i)}\right)}, \\
& Y_{3}^{n k}\left(\mathbf{P}^{(i)}\right)=\sum_{n^{\prime} \neq n, k \in \mathcal{S}_{u^{\prime} n^{\prime}}(d)} \frac{G_{\mathrm{r}_{j n}, \mathrm{~d}_{u^{\prime} n^{\prime}}}^{k}}{f_{\mathrm{d}_{u^{\prime} n^{\prime}}}^{k} t_{2}}\left(\mathbf{P}^{(i)}\right)
\end{aligned}
$$$$
Y_{4}^{n k}\left(v^{(i)}, \mathbf{P}^{(i)}\right)=\sum_{n^{\prime} \neq n, k \in \mathcal{S}_{u^{\prime} j^{\prime} n^{\prime}}(r)} v_{n^{\prime} k}^{(i)} \frac{G_{\mathrm{r}_{j n}, \mathrm{~d}_{u^{\prime} n^{\prime}}}^{k}}{f_{\mathrm{d}_{u^{\prime} n^{\prime}}, t_{2}}^{k}\left(\mathbf{P}^{(i)}\right)},
$$$$
Y_{5}^{n k}\left(\mathbf{P}^{(i)}\right)=\sum_{n^{\prime} \neq n, k \in \mathcal{S}_{u^{\prime} n^{\prime}}(d)} \frac{G_{\mathrm{s}_{n}, \mathrm{~d}_{u^{\prime} n^{\prime}}}^{k}}{f_{\mathrm{d}_{u^{\prime} n^{\prime}}, t_{2}}^{k}\left(\mathbf{P}^{(i)}\right)},
$$$$
Y_{6}^{n k}\left(v^{(i)}, \mathbf{P}^{(i)}\right)=\sum_{n^{\prime} \neq n, k \in \mathcal{S}_{u^{\prime} j^{\prime} n^{\prime}}(r)} v_{n^{\prime} k}^{(i)} \frac{G_{\mathrm{s}_{n}, \mathrm{~d}_{u^{\prime} n^{\prime}}}^{k}}{f_{\mathrm{d}_{u^{\prime} n^{\prime}}, t_{2}}\left(\mathbf{P}^{(i)}\right)} .
$$

We now consider the following theorem.

Theorem 1. $\mathbf{H}(\mathbf{P})=\left\{h_{4}^{n k}(\mathbf{P}), h_{5}^{n k}(\mathbf{P}), h_{6}^{n k}(\mathbf{P})\right\}$ satisfies the three properties of the standard interference function proposed in [35]. 
Proof. Taking equations $h_{4}^{n k}$ for example, we denote the right-hand-side functions as

$$
\begin{aligned}
& h_{4}^{n k}(\mathbf{P})=\frac{X_{1}^{n k}\left(\mathbf{P}_{0}\right)+X_{2}^{n k}\left(\mu, \mathbf{P}_{0}\right)}{\frac{\lambda_{n}}{P_{T}^{n}}+Y_{1}^{n k}(\mathbf{P})+Y_{2}^{n k}(\mu, \mathbf{P})}, \\
& Y_{1}^{n k}(\mathbf{P})=\sum_{n^{\prime} \neq n, k \in \mathcal{S}_{u^{\prime} n^{\prime}}(d)} \frac{G_{\mathrm{s}_{n}, \mathrm{~d}_{u^{\prime} n^{\prime}}}^{k}+I_{\mathrm{d}_{u^{\prime} n^{\prime}}, t_{1}}(\mathbf{P})}{\sigma^{2}}, \\
& Y_{2}^{n k}(\mu, \mathbf{P})=\sum_{n^{\prime} \neq n, k \in \mathcal{S}_{u^{\prime} j^{\prime} n^{\prime}}(r)} \mu_{n^{\prime} k} \frac{G_{\mathrm{s}_{n}, \mathrm{r}_{j^{\prime} n^{\prime}}}^{k}}{\sigma^{2}+I_{\mathrm{r}_{j^{\prime} n^{\prime}}}^{k}(\mathbf{P})} .
\end{aligned}
$$

Here, the values of $\lambda_{n}^{(i)}, \mu_{n^{\prime} k}^{(i)}, X_{1}^{n k}\left(\mathbf{P}_{0}\right)$, and $X_{2}^{n k}\left(\mu^{(i)}, \mathbf{P}_{0}\right)$ are all constant and positive. Therefore, it is obvious that $h_{4}^{n k}(\mathbf{P})>0$. Also, both $I_{\mathrm{d}_{u^{\prime} n^{\prime}}, t_{1}}^{k}(\mathbf{P})$ and $I_{\mathrm{r}_{j^{\prime} n^{\prime}}}^{k}(\mathbf{P})$ are linear functions of power variables. Thus, it is obvious that $h_{4}^{n k}(\mathbf{P})$ is a monotonic increasing function of $\mathbf{P}$, meaning that $h_{4}^{n k}(\mathbf{P})>h_{4}^{n k}\left(\mathbf{P}^{\prime}\right), \forall \mathbf{P}>\mathbf{P}^{\prime}$. Here, the inequality should be understood as element-wise. Note for all $\alpha>1$, we have

$$
\begin{aligned}
\alpha h_{4}^{n k}(\mathbf{P}) & =\frac{X_{1}^{n k}\left(\mathbf{P}_{0}\right)+X_{2}^{n k}\left(\mu, \mathbf{P}_{0}\right)}{\frac{\lambda_{n}}{\alpha P_{T}^{n}}+\frac{Y_{1}^{n k}(\mathbf{P})}{\alpha}+\frac{Y_{2}^{n k}(\mu, \mathbf{P})}{\alpha}} \\
& >\frac{X_{1}^{n k}\left(\mathbf{P}_{0}\right)+X_{2}^{n k}\left(\mu, \mathbf{P}_{0}\right)}{\frac{\lambda_{n}}{P_{T}^{n}}+\check{Y}_{1}^{n k}(\alpha, \mathbf{P})+\check{Y}_{2}^{n k}(\alpha, \mu, \mathbf{P})}
\end{aligned}
$$

where

$$
\begin{aligned}
& \check{Y}_{1}^{n k}(\alpha, \mathbf{P})=\sum_{n^{\prime} \neq n, k \in \mathcal{S}_{u^{\prime} n^{\prime}}(d)} \frac{G_{\mathrm{s}_{n}, \mathrm{~d}_{u^{\prime} n^{\prime}}}^{k}}{\sigma^{2}+\alpha I_{\mathrm{d}_{u^{\prime} n^{\prime}}, t_{1}}^{k}(\mathbf{P})}, \\
& \check{Y}_{2}^{n k}(\alpha, \mu, \mathbf{P})=\sum_{n^{\prime} \neq n, k \in \mathcal{S}_{u^{\prime} j^{\prime} n^{\prime}}(r)} \mu_{n^{\prime} k} \frac{G_{\mathrm{s}_{n}, \mathrm{r}_{j^{\prime} n^{\prime}}}^{k}}{\sigma^{2}+\alpha I_{\mathrm{r}_{j^{\prime} n^{\prime}}}^{k}(\mathbf{P})} .
\end{aligned}
$$

According to the expression of $I_{\mathrm{d}_{u n}, t_{1}}^{k}(\mathbf{P})$ and $I_{\mathrm{r}_{j n}}^{k}(\mathbf{P})$, it is obvious that $\alpha I_{\mathrm{d}_{u n}, t_{1}}^{k}(\mathbf{P})=I_{\mathrm{d}_{u n}, t_{1}}^{k}(\alpha \mathbf{P})$ and $\alpha I_{\mathrm{r}_{j n}}^{k}(\mathbf{P})=$ $I_{\mathrm{r}_{j n}}^{k}(\alpha \mathbf{P})$. Thus, we have

$$
\alpha h_{4}^{n k}(\mathbf{P})>h_{4}^{n k}(\alpha \mathbf{P}), \forall \alpha>1, n, k
$$

Similarly, we have $h_{5}^{n k}(\mathbf{P})>0, \alpha h_{5}^{n k}(\mathbf{P})>$ $h_{5}^{n k}(\alpha \mathbf{P}), \forall \alpha>1, n, k \in \mathcal{S}_{u j n}(r)$, and $h_{6}^{n k}(\mathbf{P})>$ $0, \alpha h_{6}^{n k}(\mathbf{P})>h_{6}^{n k}(\alpha \mathbf{P}), \forall \alpha>1, n, k \in \mathcal{S}_{u n}(d)$. Both $h_{5}^{n k}(\mathbf{P})$ and $h_{6}^{n k}(\mathbf{P})$ are monotonic increasing functions of $\mathbf{P}$.
Note that each element of $\mathbf{H}(\mathbf{P})$ satisfies the three properties (positivity, monotonicity, scalability) of the standard interference function defined in [35]. Thus, $\mathbf{H}(\mathbf{P})$ is a standard interference function.

Thanks to the Theorems 1 and 2 in [35], the standard interference function $\mathbf{H}(\mathbf{P})$ has a unique fixed point solution, which can be found iteratively as follows:

$$
\begin{aligned}
& P_{\mathrm{s}_{n}, t_{1}}^{k,\left(i, i^{\prime}+1\right)}=h_{4}^{n k}\left(\lambda_{n}^{(i)}, \mu^{(i)}, \mathbf{P}^{\left(i, i^{\prime}\right)}\right), \forall n, k, i, \\
& P_{\mathrm{r}_{j n}}^{k,\left(i, i^{\prime}+1\right)}=h_{5}^{n k}\left(\lambda_{n}^{(i)}, v^{(i)}, \mathbf{P}^{\left(i, i^{\prime}\right)}\right), \forall n, k \in \mathcal{S}_{u j n}(r), i, \\
& P_{\mathrm{s}_{n}, t_{2}}^{k,\left(i, i^{\prime}+1\right)}=h_{6}^{n k}\left(\lambda_{n}^{(i)}, v^{(i)}, \mathbf{P}^{\left(i, i^{\prime}\right)}\right), \forall n, k \in \mathcal{S}_{u n}(d), i .
\end{aligned}
$$

Let us denote

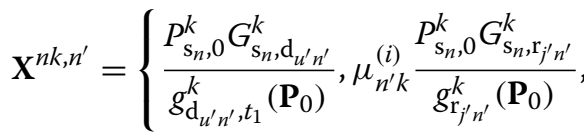

$$
\begin{aligned}
& v_{n^{\prime} k}^{(i)} \frac{P_{\mathrm{r}_{j n}, 0}^{k} G_{\mathrm{r}_{j n}, \mathrm{~d}_{u^{\prime} n^{\prime}}}^{k}}{g_{\mathrm{d}_{u^{\prime} n^{\prime}}, t_{2}}^{k}\left(\mathbf{P}_{0}\right)}, \frac{P_{\mathrm{r}_{j n}, 0}^{k} G_{\mathrm{r}_{j n}, \mathrm{~d}_{u^{\prime} n^{\prime}}}^{k}}{g_{\mathrm{d}_{u^{\prime} n^{\prime}}, t_{2}}^{k}\left(\mathbf{P}_{0}\right)}, \\
& \left.v_{n^{\prime} k}^{(i)} \frac{P_{\mathrm{s} 1_{n}, 0}^{k} G_{\mathrm{s}_{n}, \mathrm{~d}_{u^{\prime} n^{\prime}}}^{k}}{g_{\mathrm{d}_{u^{\prime} n^{\prime}}, t_{2}}^{k}\left(\mathbf{P}_{0}\right)}, \frac{P_{\mathrm{s}_{n}, 0}^{k} G_{\mathrm{s}_{n}, \mathrm{~d}_{u^{\prime} n^{\prime}}}^{k}}{g_{\mathrm{d}_{u^{\prime} n^{\prime}}, t_{2}}^{k}\left(\mathbf{P}_{0}\right)}\right\} \text {, } \\
& \mathbf{Y}^{n k, n^{\prime}}=\left\{\frac{1}{f_{\mathrm{d}_{u^{\prime} n^{\prime}, t_{1}}}^{k}\left(\mathbf{P}^{(i)}\right)}, \frac{\mu_{n^{\prime} k}^{(i)}}{f_{\mathrm{r}_{j^{\prime} n^{\prime}}}^{k}\left(\mathbf{P}^{(i)}\right)}, \frac{v_{n^{\prime} k}^{(i)}}{f_{\mathrm{d}_{u^{\prime} n^{\prime}, t_{2}}^{k}\left(\mathbf{P}^{(i)}\right)}^{k}},\right. \\
& \left.\frac{G_{\mathrm{r}_{j n}, \mathrm{~d}_{u^{\prime} n^{\prime}}}^{k}}{f_{\mathrm{d}_{u^{\prime} n^{\prime}}, t_{2}}^{k}\left(\mathbf{P}^{(i)}\right)}, v_{n^{\prime} k}^{(i)} \frac{G_{\mathrm{s}_{n}, \mathrm{~d}_{u^{\prime} n^{\prime}}}^{k}}{f_{\mathrm{d}_{u^{\prime} n^{\prime}, t_{2}}^{k}\left(\mathbf{P}^{(i)}\right)}^{k}}, \frac{G_{\mathrm{s}_{n}, \mathrm{~d}_{u^{\prime} n^{\prime}}}^{k}}{f_{\mathrm{d}_{u^{\prime} n^{\prime}, t_{2}}}^{k}\left(\mathbf{P}^{(i)}\right)}\right\} \text {. }
\end{aligned}
$$

As we assume that each receiver in each cell can estimate the received power from each of the interfering stations (either the BS or a RS of an interfering cell), both $\mathbf{X}^{n k, n^{\prime}}$ and $\mathbf{Y}^{n k, n^{\prime}}$ can be calculated locally in each interfering cell $n^{\prime}$ for a considered cell $n$. We further assume that the central controller (CC) has access to the estimates of all the cross gains $\left(G_{\mathrm{s}_{n}, \mathrm{~d}_{u^{\prime} n^{\prime}}}^{k}, G_{\mathrm{s}_{n}, \mathrm{r}_{j^{\prime} n^{\prime}}}^{k}, G_{\mathrm{r}_{j n}, \mathrm{~d}_{u^{\prime} n^{\prime}}}^{k}\right)$ between two different cells. Thus, $\mathbf{X}^{n}=\left\{X_{1}^{n k}, X_{2}^{n k}, X_{3}^{n k}, X_{4}^{n k}, X_{5}^{n k}, X_{6}^{n k}\right\}$ and $\mathbf{Y}^{n}=$ $\left\{Y_{1}^{n k}, Y_{2}^{n k}, Y_{3}^{n k}, Y_{4}^{n k}, Y_{5}^{n k}, Y_{6}^{n k}\right\}$ can be calculated in the $\mathrm{CC}$ with the uploaded values of $\mathbf{X}^{n k, n^{\prime}}$ and $\mathbf{Y}^{n k, n^{\prime}}$ from each cell. Finally, the SCLD PA algorithm is described in Algorithm 2. Note that, through estimation of the received power and message passing, the SCLD PA algorithm is semi-distributed with the help of a CC. 


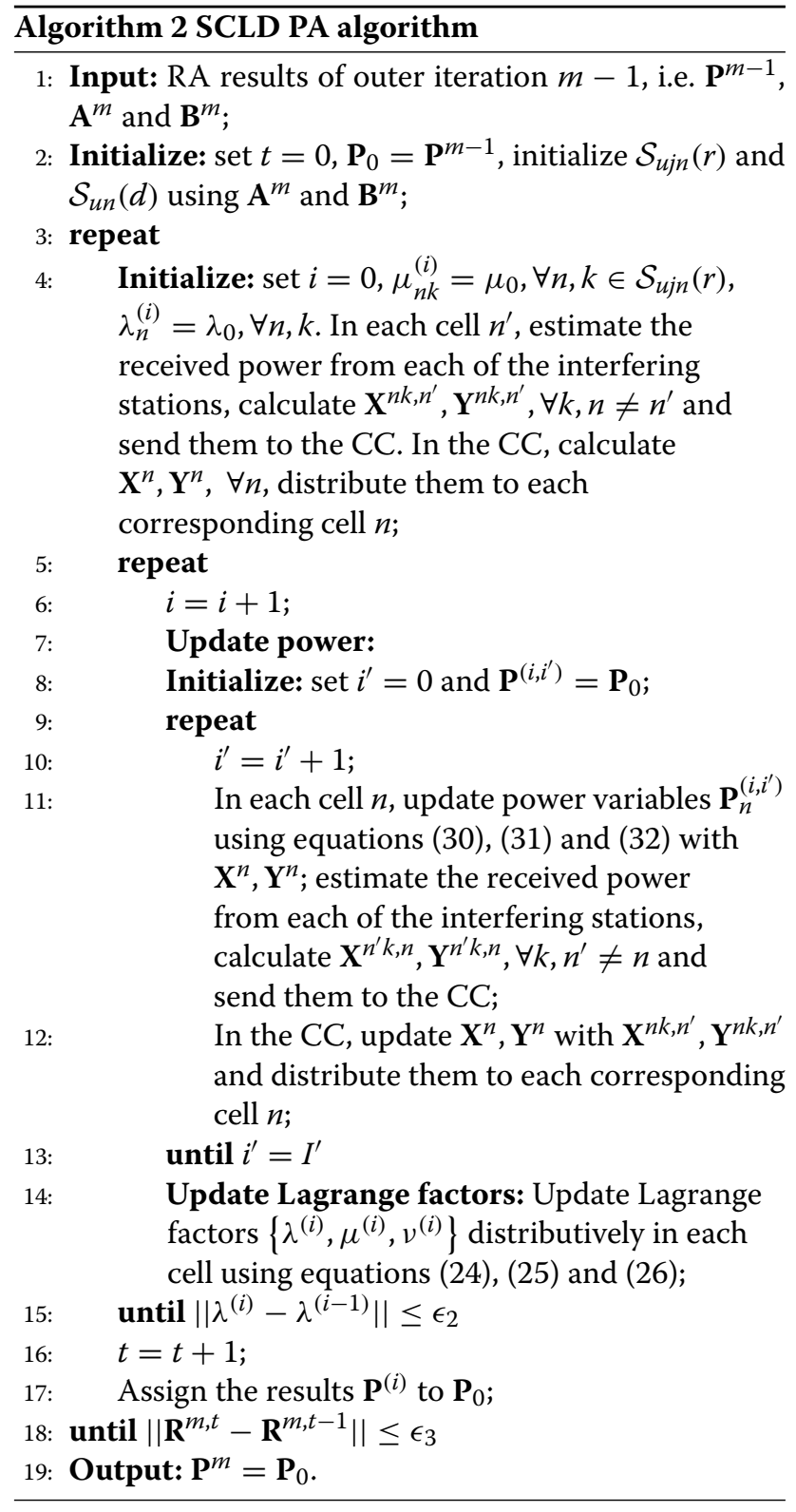

\section{Modified IWF algorithm}

In order to solve the formulated problem autonomously in each cell, we now propose the modified iterative waterfilling (M-IWF) algorithm, which is inspired by the noncooperative game-theoretic approach (named as the IWF algorithm) proposed by [36] in DSL systems. In there, with the interfering users' power values fixed, each user measures the interference plus noise levels locally and allocates its power greedily to maximize its own rate. Although its convergence is guaranteed under limited cases where the crosstalk interferences are weak, the IWF algorithm has become a popular candidate for multi-cell power allocation due to its interesting trade-off between the complexity and the performance.
In this section, we mimic the idea of the IWF algorithm and formulate a competitive game defining each cell as a player, which optimizes its own utility function (meaning the sum rate of this cell) given the RA results of the other cells. In each cell, both the indicators and the power variables are optimized which makes the local problem more interesting than that of [36]. The CCI received by the cell under consideration is fixed and treated as an additive noise. The received power of the interference and noise at each subcarrier $k$ of each receiver (either a RS or a MS) in cell $n$ is a constant. Specifically, $f_{\mathrm{r}_{j n}}^{k}=\sigma^{2}+\sum_{n^{\prime}, n^{\prime} \neq n} P_{\mathrm{s}_{n^{\prime}}, t_{1}}^{k} G_{\mathrm{s}_{n^{\prime}}, \mathrm{r}_{j n}}^{k}, \forall j, k, f_{\mathrm{d}_{u n}, t_{2}}^{k}=\sigma^{2}+$ $\sum_{n^{\prime}=1, n^{\prime} \neq n}^{N} P_{\mathrm{s}_{n^{\prime}}, t_{2}}^{k} G_{\mathrm{s}_{n^{\prime}}, \mathrm{d}_{u n}}^{k}+\sum_{n^{\prime}=1, n^{\prime} \neq n}^{N} \sum_{j^{\prime}=1}^{J} P_{\mathrm{r}_{j^{\prime} n^{\prime}}}^{k} G_{\mathrm{r}_{j^{\prime} n^{\prime}}, \mathrm{d}_{u n}}^{k}, \forall u, k$ and $f_{\mathrm{d}_{u n}, t_{1}}^{k}=\sigma^{2}+\sum_{n^{\prime}=1, n^{\prime} \neq n}^{N} P_{\mathrm{s}_{n^{\prime}}, t_{1}}^{k} G_{\mathrm{s}_{n^{\prime}}, \mathrm{d}_{u n}}^{k}, \forall u, k$. Thus, the achievable sum rate of cell $n$ is denoted as follows:

$$
\begin{aligned}
R_{n}\left(\mathbf{P}_{n}, \mathbf{A}_{n}, \mathbf{B}_{n}\right)= & \sum_{u=1}^{U} \sum_{k=1}^{K} a_{u n}^{k} R_{u n, 1}^{k}\left(\mathbf{P}_{\mathbf{n}}^{\mathbf{k}}\right) \\
& +\sum_{u=1}^{U} \sum_{j=1}^{J} \sum_{k=1}^{K} b_{u j n}^{k} R_{u j n, 2}^{k}\left(\mathbf{P}_{\mathbf{n}}^{\mathbf{k}}\right) .
\end{aligned}
$$

where $\mathbf{A}_{n}=\left\{a_{u n^{\prime}}^{k} \mid n^{\prime}=n\right\}, \mathbf{B}_{n}=\left\{b_{u j n^{\prime}}^{k} \mid n^{\prime}=n\right\}, R_{u n, 1}^{k}=\ln$ $\left(1+\Upsilon_{\mathrm{d}_{u n}, t_{1}}^{k}\right)+\ln \left(1+\Upsilon_{\mathrm{d}_{u n}, t_{2}}^{k}\right), R_{u j n, 2}^{k}=\min \left(\ln \left(1+\Gamma_{\mathrm{r}_{j n}}^{k}\right)\right.$, $\left.\ln \left(1+\Gamma_{\mathrm{d}_{u n}, t_{2}}^{k}\right)\right), \quad \Upsilon_{\mathrm{d}_{u n}, t_{1}}^{k}=P_{\mathrm{s}_{n}, t_{1}}^{k} \Lambda_{\mathrm{s}_{n}, \mathrm{~d}_{u n}}^{k, t_{1}}, \Upsilon_{\mathrm{d}_{u n}, t_{2}}^{k}=$ $P_{\mathrm{s}_{n}, t_{2}}^{k} \Lambda_{\mathrm{s}_{n}, \mathrm{~d}_{u n}}^{k, t_{2}}, \Gamma_{\mathrm{r}_{j n}}^{k}=P_{\mathrm{s}_{n}, t_{1}}^{k} \Lambda_{\mathrm{s}_{n}, \mathrm{r}_{j n}}^{k}, \Gamma_{\mathrm{d}_{u n}, t_{2}}^{k}=P_{\mathrm{r}_{j n}}^{k} \Lambda_{\mathrm{r}_{j n}, \mathrm{~d}_{u n}}^{k}$,

$$
\begin{aligned}
\Lambda_{\mathrm{s}_{n}, \mathrm{~d}_{u n}}^{k, t_{1}} & =\frac{G_{\mathrm{s}_{n}, \mathrm{~d}_{u n}}^{k}}{f_{\mathrm{d}_{u n}, t_{1}}^{k}} \\
\Lambda_{\mathrm{s}_{n}, \mathrm{~d}_{u n}}^{k, t_{2}} & =\frac{G_{\mathrm{s}_{n}, \mathrm{~d}_{u n}}^{k}}{f_{\mathrm{d}_{u n}, t_{2}}^{k}}, \\
\Lambda_{\mathrm{s}_{n}, \mathrm{r}_{j n}}^{k} & =\frac{G_{\mathrm{s}_{n}, \mathrm{r}_{j n}}^{k}}{f_{\mathrm{r}_{j n}}^{k}}, \\
\Lambda_{\mathrm{r}_{j n}, \mathrm{~d}_{u n}}^{k} & =\frac{G_{\mathrm{r}_{j n}, \mathrm{~d}_{u n}}^{k}}{f_{\mathrm{d}_{u n}, t_{2}}^{k}} .
\end{aligned}
$$


The local problem of cell $n$ can be formulated as the following:

$$
\begin{aligned}
& \max _{\mathbf{P}_{n}, \mathbf{A}_{n}, \mathbf{B}_{n}} R_{n}\left(\mathbf{P}_{n}, \mathbf{A}_{n}, \mathbf{B}_{n}\right) \\
& \text { s.t. } \\
& C 1^{\prime}:\left(\sum_{u=1}^{U} a_{u n}^{k}+\sum_{u=1}^{U} \sum_{j=1}^{J} b_{u j n}^{k}\right) \leq 1, \forall k, \\
& C 2^{\prime}: a_{u n}^{k} \in\{0,1\}, b_{u j n}^{k} \in\{0,1\}, \forall u, j, k, \\
& C 3^{\prime}: \sum_{k=1}^{K} P_{\mathrm{s}_{n}, t_{1}}^{k}+\sum_{k=1}^{K} P_{\mathrm{s}_{n}, t_{2}}^{k}+\sum_{j=1}^{J} \sum_{k=1}^{K} P_{\mathrm{r}_{j n}}^{k} \leq P_{t, n}, \\
& C 4^{\prime}: P_{\mathrm{s}_{n}, t_{1}}^{k} \geq 0, P_{\mathrm{s}_{n}, t_{2}}^{k} \geq 0, P_{\mathrm{r}_{j n}}^{k} \geq 0, \forall j, k \\
& C 5^{\prime}: P_{\mathrm{s}_{n}, t_{1}}^{k}=F\left(P_{\mathrm{s}_{n}, t_{1}}^{k},\left(\sum_{u=1}^{U} a_{u n}^{k}+\sum_{u=1}^{U} \sum_{j=1}^{J} b_{u j n}^{k}\right)\right), \forall k, \\
& C 7^{\prime}: P_{\mathrm{r}_{j n}}^{k}=F\left(P_{\mathrm{r}_{j n},}^{k} \sum_{u=1}^{U} b_{u j n}^{k}\right), \forall j, k . \\
& P_{\mathrm{s}_{n}, t_{2}}^{k}=F\left(P_{\mathrm{s}_{n}, t_{2}}^{k}, \sum_{u=1}^{U} a_{u n}^{k}\right), \forall k,
\end{aligned}
$$

Note that, in the case when $f_{\mathrm{d}_{u n}, t_{1}}^{k}=f_{\mathrm{d}_{u n}, t_{2}}^{k}$, the optimal RA algorithm proposed in Section 3 of [16] can be applied here with simple extension. However, when $f_{\mathrm{d}_{u n}, t_{1}}^{k} \neq f_{\mathrm{d}_{u n}, t_{2}}^{k}$, the optimization problem becomes more interesting and the algorithm of [16] can not be applied here easily. To find the optimal RA result for the formulated problem, we will first propose the optimal PA algorithm followed by the optimal MSSA algorithm. To obtain the optimal PA result, we now propose two theorems as follows:

Theorem 2. For a relayed subcarrier $k$ in cell $n$, the DF constraint is active at the optimum point of problem (38), meaning $\ln \left(1+\Gamma_{\mathrm{r}_{j n}}^{k}\right)=\ln \left(1+\Gamma_{\mathrm{d}_{u n}, t_{2}}^{k}\right)$.

Proof. We assume that the optimal RA scheme is obtained, leading to the optimal sum rate. For a relayed subcarrier $k$ in cell $n$, the optimal MS $u$ and RS $j$ are fixed. The optimal BS power and the optimal RS power are denoted as $P_{\mathrm{S}_{n}, t_{1}}^{k, \text { opt }}$ and $P_{\mathrm{r}_{j n}}^{k, o p t}$, respectively. Then we have

$$
\begin{aligned}
& \Gamma_{\mathrm{r}_{j n}}^{k}=P_{\mathrm{s}_{n}, t_{1}}^{k, o p t} \Lambda_{\mathrm{s}_{n}, \mathrm{r}_{j n}}^{k}, \\
& \Gamma_{\mathrm{d}_{u n}, t_{2}}^{k}=P_{\mathrm{r}_{j n}}^{k, o p t} \Lambda_{\mathrm{r}_{j n}, \mathrm{~d}_{u n}}^{k} .
\end{aligned}
$$

If $\Gamma_{\mathrm{r}_{j n}}^{k}>\Gamma_{\mathrm{d}_{u n}, t_{2}}^{k}$, then $R_{u j n, 2}^{k}$ is determined only by $\Gamma_{\mathrm{d}_{u n}, t_{2}}^{k}$. Note that both $\Lambda_{\mathrm{s}_{n}, \mathrm{r}_{j n}}^{k}$ and $\Lambda_{\mathrm{r}_{j n}, \mathrm{~d}_{u n}}^{k}$ are constants. Thus, $R_{u j n, 2}^{k}$ is determined only by $P_{\mathrm{r}_{j n}}^{k, o p t}$. In this case, we can still improve the sum rate $R_{n}$ by borrowing some power from $P_{\mathrm{s}_{n}, t_{1}}^{k, \text { opt }}$ to $P_{\mathrm{r}_{j n}}^{k, o p t}$ while keeping $\Gamma_{\mathrm{r}_{j_{n}}}^{k}>\Gamma_{\mathrm{d}_{u n}, t_{2}}^{k}$. This obviously contradicts the previous optimal sum rate assumption.

Similarly, if $\Gamma_{\mathrm{r}_{j n}}^{k}<\Gamma_{\mathrm{d}_{u n}, t_{2}}^{k}$, the optimal sum rate $R_{n}$ can also be improved by borrowing some power from $P_{\mathrm{r}_{j n}}^{k, o p t}$ to $P_{\mathrm{s}_{n}, t_{1}}^{k, \text { opt }}$ while keeping the inequality. This again contradicts the previous optimal sum rate assumption. Therefore, at the optimal point, $\Gamma_{\mathrm{r}_{j n}}^{k}=\Gamma_{\mathrm{d}_{u n}, t_{2}}^{k}$ and $\ln \left(1+\Gamma_{\mathrm{r}_{j n}}^{k}\right)=\ln \left(1+\Gamma_{\mathrm{d}_{u n}, t_{2}}^{k}\right)$. This concludes the proof of Theorem 2.

According to Theorem 2, we now have $\Gamma_{\mathrm{r}_{j n}}^{k}=\Gamma_{\mathrm{d}_{u n}, t_{2}}^{k}$, which means

$$
\begin{aligned}
& P_{\mathrm{s}_{n}, t_{1}}^{k}=P_{\mathrm{r}_{j n}}^{k} \alpha_{u n}^{k}=P_{n}^{k} \frac{\alpha_{u n}^{k}}{1+\alpha_{u n}^{k}}, \quad \forall k \in S_{n}(r) \\
& \alpha_{u n}^{k}=\frac{\Lambda_{\mathrm{r}_{j n}, \mathrm{~d}_{u n}}^{k}}{\Lambda_{\mathrm{s}_{n}, \mathrm{r}_{j n}}^{k}}, \quad \forall k \in S_{n}(r) \\
& P_{n}^{k}= \begin{cases}P_{\mathrm{s}_{n}, t_{1}}^{k}+P_{\mathrm{s}_{n}, t_{2}}^{k} & k \in S_{n}(d) \\
P_{\mathrm{s}_{n}, t_{1}}^{k}+P_{\mathrm{r}_{j n}}^{k} & k \in S_{n}(r)\end{cases}
\end{aligned}
$$

where $S_{n}(d)$ denotes the set of subcarriers with direct mode in cell $n, S_{n}(r)$ denotes the set of subcarriers with relay-aided mode in cell $n$.

Theorem 3. For a direct mode subcarrier $k$ in cell $n$, at the optimum point of problem (38), we have

$$
\begin{aligned}
& P_{\mathrm{s}_{n}, t_{1}}^{k, \text { opt }}=\frac{P_{n}^{k, \text { opt }}}{2}+\frac{\Lambda_{\mathrm{s}_{n}, \mathrm{~d}_{u n}}^{k, t_{1}}-\Lambda_{\mathrm{s}_{n}, \mathrm{~d}_{u n}}^{k, t_{2}}}{2 \Lambda_{\mathrm{s}_{n}, \mathrm{~d}_{u n}}^{k, t_{1}} \Lambda_{\mathrm{s}_{n}, \mathrm{~d}_{u n}}^{k, t_{2}}}, \\
& P_{\mathrm{s}_{n}, t_{2}}^{k, o p t}=\frac{P_{n}^{k, o p t}}{2}+\frac{\Lambda_{\mathrm{s}_{n}, \mathrm{~d}_{u n}}^{k, t_{2}}-\Lambda_{\mathrm{s}_{n}, \mathrm{~d}_{u n}}^{k, t_{1}}}{2 \Lambda_{\mathrm{s}_{n}, \mathrm{~d}_{u n}}^{k, t_{1}} \Lambda_{\mathrm{s}_{n}, \mathrm{~d}_{u n}}^{k, t_{2}}} .
\end{aligned}
$$
(38).

Here, $P_{n}^{k, \text { opt }}$ denotes the optimal value of $P_{n}^{k}$ for problem

Proof. Let us introduce the following problem:

$$
\begin{aligned}
& \max _{P_{\mathrm{s}_{n}, t_{1}}^{k}, P_{\mathrm{s}_{n}, t_{2}}^{k}} R_{u n, 1}^{k}\left(P_{\mathrm{s}_{n}, t_{1}}^{k}, P_{\mathrm{s}_{n}, t_{2}}^{k}\right) \\
& \text { s.t. } P_{\mathrm{s}_{n}, t_{1}}^{k}+P_{\mathrm{s}_{n}, t_{2}}^{k}=P_{n}^{k, o p t},
\end{aligned}
$$


where $R_{u n, 1}^{k}=\ln \left(1+P_{\mathrm{s}_{n}, t_{1}}^{k} \Lambda_{\mathrm{s}_{n}, \mathrm{~d}_{u n}}^{k, t_{1}}\right)+\ln \left(1+P_{\mathrm{s}_{n}, t_{2}}^{k} \Lambda_{\mathrm{s}_{n}, \mathrm{~d}_{u n}}^{k, t_{2}}\right)$. By introducing a Lagrange multiplier $\zeta$, the Lagrange function of problem (46) is denoted as

$$
L=R_{u n, 1}^{k}-\zeta\left(P_{\mathrm{s}_{n}, t_{1}}^{k}+P_{\mathrm{s}_{n}, t_{2}}^{k}-P_{n}^{k, o p t}\right)
$$

After taking the derivative of $L$ w.r.t $P_{\mathrm{s}_{n}, t_{1}}^{k}$ and setting it to 0 , we certainly have $\frac{\Lambda_{\mathrm{s}_{n}, \mathrm{~d} u n}^{k, t_{1}}}{1+\Lambda_{\mathrm{s}_{n}, \mathrm{~d}_{u n}}^{k, t_{\mathrm{s}_{n}, t_{1}}} P^{k}}=\zeta$. Similarly, we have $\frac{\Lambda_{\mathrm{s}_{n}, \mathrm{~d}_{u n}}^{k, t_{2}}}{1+\Lambda_{\mathrm{s}_{n}, \mathrm{~d}_{u n}}^{k, d_{\mathrm{s}_{n}, t_{2}}} P^{k}}=\zeta$. Considering the sum power constraint, we can easily calculate the optimal values for $P_{\mathrm{s}_{n}, t_{1}}^{k}$ and $P_{\mathrm{s}_{n}, t_{2}}^{k}$ of problem (46) as $\frac{P_{n}^{k, o p t}}{2}+\frac{\Lambda_{\mathrm{s}_{n}, \mathrm{~d} u n}^{k, t_{1}}-\Lambda_{\mathrm{s}_{n}, \mathrm{~d} u n}^{k, t_{2}}}{2 \Lambda_{\mathrm{s}_{n}, \mathrm{~d}_{u n}}^{k, t_{1}} \Lambda_{\mathrm{s}_{n}, \mathrm{~d} \mathrm{~d}_{u n}}^{k, t_{2}}}$ and $\frac{P_{n}^{k, o p t}}{2}+\frac{\Lambda_{\mathrm{s}_{n}, \mathrm{~d}_{u n}}^{k, t_{2}}-\Lambda_{\mathrm{s}_{n}, \mathrm{~d}_{u n}}^{k, t_{1}}}{2 \Lambda_{\mathrm{s}_{n}, \mathrm{~d}_{u n}}^{k, t_{1}} \Lambda_{\mathrm{s}_{n}, \mathrm{~d}_{u n}}^{k}}$, respectively.

Thus, for a direct mode subcarrier $k$ in cell $n$, at the optimum point of problem (38), we have $P_{\mathrm{S}_{n}, t_{1}}^{k, \text { opt }}=\frac{P_{n}^{k, \text { opt }}}{2}+$ $\frac{\Lambda_{\mathrm{s}_{n}, \mathrm{~d} u n}^{k, t_{u}}-\Lambda_{\mathrm{s}_{n}, \mathrm{~d}_{u n}}^{k, t_{2}}}{2 \Lambda_{\mathrm{s}_{n}, \mathrm{~d}_{u n}}^{k, t_{n}} \Lambda_{\mathrm{s}_{n}, \mathrm{~d}_{u n}}^{k, t_{2}}}$ and $P_{\mathrm{s}_{n}, t_{2}}^{k, o p t}=\frac{P_{n}^{k, o p t}}{2}+\frac{\Lambda_{\mathrm{s}_{n}, \mathrm{~d} u n}^{k, t_{2}}-\Lambda_{\mathrm{s}_{n}, \mathrm{~d}_{u n}}^{k, t_{u n}}}{2 \Lambda_{\mathrm{s}_{n}, t_{n}}^{k, \mathrm{~d}_{u n}} \Lambda_{\mathrm{s}_{n}, \mathrm{~d}_{u n}}^{k, t_{u}}}$. Otherwise, we can still improve $R_{u n, 1}^{k}$ and $R_{n}$. This concludes the proof of Theorem 3 .

According to Theorem 2, the rate of a relayed subcarrier $\left(R_{u j n, 2}^{k}\right)$ when allocated to a MS $\mathrm{d}_{u n}$ with the help of a RS $\mathrm{r}_{j n}$ in cell $n$ is denoted as

$$
\ln \left(1+P_{n}^{k} \frac{\Lambda_{\mathrm{s}_{n}, \mathrm{r}_{j n}}^{k} \alpha_{u n}^{k}}{1+\alpha_{u n}^{k}}\right)
$$

Also according to Theorem 3, the rate of a direct mode subcarrier $\left(R_{u n, 1}^{k}\right)$ when allocated to a MS $\mathrm{d}_{u n}$ in cell $n$ is denoted as

$$
\begin{aligned}
& \ln \left(1+\frac{P_{n}^{k, o p t} \Lambda_{\mathrm{s}_{n}, \mathrm{~d}_{u n}}^{k, t_{1}}}{2}+\frac{\Lambda_{\mathrm{s}_{n}, \mathrm{~d}_{u n}}^{k, t_{1}}-\Lambda_{\mathrm{s}_{n}, \mathrm{~d}_{u n}}^{k, t_{2}}}{2 \Lambda_{\mathrm{s}_{n}, \mathrm{~d}_{u n}}^{k, t_{2}}}\right) \\
& +\ln \left(1+\frac{P_{n}^{k, o p t} \Lambda_{\mathrm{s}_{n}, \mathrm{~d}_{u n}}^{k, t_{2}}}{2}+\frac{\Lambda_{\mathrm{s}_{n}, \mathrm{~d}_{u n}}^{k, t_{2}}-\Lambda_{\mathrm{s}_{n}, \mathrm{~d}_{u n}}^{k, t_{1}}}{2 \Lambda_{\mathrm{s}_{n}, \mathrm{~d}_{u n}}^{k, t_{1}}}\right),
\end{aligned}
$$

which can be further denoted as

$$
\begin{gathered}
\ln \left(1+P_{n}^{k} \frac{\Lambda_{\mathrm{s}_{n}, \mathrm{~d}_{u n}}^{k, t_{1}}+\Lambda_{\mathrm{s}_{n}, \mathrm{~d}_{u n}}^{k, t_{2}}}{2}+\left(P_{n}^{k}\right)^{2} \frac{\Lambda_{\mathrm{s}_{n}, \mathrm{~d}_{u n}}^{k, t_{1}} \Lambda_{\mathrm{s}_{n}, \mathrm{~d}_{u n}}^{k, t_{2}}}{4}\right. \\
\left.+\frac{\left(\Lambda_{\mathrm{s}_{n}, \mathrm{~d}_{u n}}^{k, t_{1}}-\Lambda_{\mathrm{s}_{n}, \mathrm{~d}_{u n}}^{k, t_{2}}\right)^{2}}{4 \Lambda_{\mathrm{s}_{n}, \mathrm{~d}_{u n}}^{k, t_{1}} \Lambda_{\mathrm{s}_{n}, \mathrm{~d}_{u n}}^{k, t_{2}}}\right)
\end{gathered}
$$

Let us first propose the PA algorithm with the MSSA results fixed. By introducing a Lagrange multiplier $\gamma_{n}$, the Lagrange function is denoted as

$$
L_{n}=R_{n}-\gamma_{n}\left(\sum_{k=1}^{N_{t}} P_{n}^{k}-P_{t}^{n}\right) .
$$

For a direct mode subcarrier $k$, after taking the derivative of $L_{n}$ w.r.t. $P_{n}^{k}, k \in S_{n}(d)$ and setting $-4 \frac{\partial L_{n}}{\partial P_{n}^{k}}=0$, we have $F_{2}\left(P_{n}^{k}\right)=0$, where $F_{2}\left(P_{n}^{k}\right)$ is denoted as follows:

$$
\begin{aligned}
& \left(P_{n}^{k}\right)^{2} \gamma_{n} \Lambda_{\mathrm{s}_{n}, \mathrm{~d}_{u n}}^{k, t_{1}} \Lambda_{\mathrm{s}_{n}, \mathrm{~d}_{u n}}^{k, t_{2}} \\
& +P_{n}^{k}\left[2 \gamma_{n}\left(\Lambda_{\mathrm{s}_{n}, \mathrm{~d}_{u n}}^{k, t_{1}}+\Lambda_{\mathrm{s}_{n}, \mathrm{~d}_{u n}}^{k, t_{2}}\right) 2 \Lambda_{\mathrm{s}_{n}, \mathrm{~d}_{u n}}^{k, t_{1}} \Lambda_{\mathrm{s}_{n}, \mathrm{~d}_{u n}}^{k, t_{2}}\right] \\
& +\frac{\gamma_{n}\left(\Lambda_{\mathrm{s}_{n}, \mathrm{~d}_{u n}}^{k, t_{1}}+\Lambda_{\mathrm{s}_{n}, \mathrm{~d}_{u n}}^{k, t_{2}}\right)^{2}}{\Lambda_{\mathrm{s}_{n}, \mathrm{~d}_{u n}}^{k, t_{1}} \Lambda_{\mathrm{s}_{n}, \mathrm{~d}_{u n}}^{k, t_{2}}}-2\left(\Lambda_{\mathrm{s}_{n}, \mathrm{~d}_{u n}}^{k, t_{1}}+\Lambda_{\mathrm{s}_{n}, \mathrm{~d}_{u n}}^{k, t_{2}}\right) .
\end{aligned}
$$

After some mathematical calculations, the discriminant of $F_{2}\left(P_{n}^{k}\right)$ is denoted as $4\left(\Lambda_{\mathrm{s}_{n}, \mathrm{~d}_{u n}}^{k, t_{n}} \Lambda_{\mathrm{s}_{n}, \mathrm{~d}_{u n}}^{k, t_{2}}\right)^{2}$. The roots are denoted as $\frac{2}{\gamma_{n}}-\frac{\Lambda_{\mathrm{s}_{n}, \mathrm{~d}_{u n}}^{k, t_{1}}+\Lambda_{\mathrm{s}_{n}, \mathrm{~d}_{u n}}^{k, t_{2}}}{\Lambda_{\mathrm{s}_{n}, \mathrm{~d}_{u n}}^{k, t_{1}} \Lambda_{\mathrm{s}_{n}, \mathrm{~d}_{u n}}^{k, \mathrm{~d}_{u}}}$ and $-\frac{\Lambda_{\mathrm{s}_{n}, \mathrm{~d}_{u n}}^{k, t_{1}}+\Lambda_{\mathrm{s}_{n}, \mathrm{~d}_{u n}}^{k, t_{2}}}{\Lambda_{\mathrm{s}_{n}, \mathrm{~d}_{u n}}^{k, t_{1}} \Lambda_{\mathrm{s}_{n}, \mathrm{~d}_{u n}}^{k, \mathrm{~d}_{u}}}$. Note that $-\frac{\Lambda_{\mathrm{s}_{n}, \mathrm{~d}_{u n}}^{k, t_{1}}+\Lambda_{\mathrm{s}_{n}, \mathrm{~d}_{u n}}^{k, t_{2}}}{\Lambda_{\mathrm{s}_{n}, \mathrm{~d}_{u n}}^{k, \mathrm{~d}_{2}} \Lambda_{\mathrm{s}_{n}, \mathrm{~d}_{u n}}^{\mathrm{s}_{n}, \mathrm{~d}_{u n}}}<0$. Thus by setting $F_{2}\left(P_{n}^{k}\right)$ to 0 , the allocated total power for the direct mode subcarrier $k$ in cell $n$ is given by

$$
P_{n}^{k}=\left[\frac{2}{\gamma_{n}}-\frac{\Lambda_{\mathrm{s}_{n}, \mathrm{~d}_{u n}}^{k, \mathrm{t}_{1}}+\Lambda_{\mathrm{s}_{n}, \mathrm{~d}_{u n}}^{k, t_{2}}}{\Lambda_{\mathrm{s}_{n}, \mathrm{~d}_{u n}}^{k, t_{1}} \Lambda_{\mathrm{s}_{n}, \mathrm{~d}_{u n}}^{k, t_{2}}}\right]_{+}
$$

where $[.]_{+}$stands for $\max [0,$.$] .$

For a relayed subcarrier $k$, after taking the derivative of $L_{n}$ w.r.t. $P_{n}^{k}, k \in S_{n}(r)$ and setting it to 0 , we obtain

$$
P_{n}^{k}=\left[\frac{1}{\gamma_{n}}-\frac{1+\alpha_{u n}^{k}}{\Lambda_{\mathrm{s}_{n}, \mathrm{r}_{j n}}^{k} \alpha_{u n}^{k}}\right]_{+}
$$

The derivations show that the constrained optimization problem can be solved using the water-filling algorithm, applied to a water container built either from $\frac{\Lambda_{\mathrm{s}_{n}, \mathrm{~d} u n}^{k, t_{1}}+\Lambda_{\mathrm{s}_{n}, \mathrm{~d}_{u n}}^{k, t_{2}}}{\Lambda_{\mathrm{s}_{n}, \mathrm{~d}_{u n}}^{k, t_{n}} \Lambda_{\mathrm{s}_{n}, \mathrm{~d}_{u n}}^{k, \mathrm{~d}_{u}}}$ or from $\frac{1+\alpha_{u n}^{k}}{\Lambda_{\mathrm{s}_{n}, \mathrm{r}_{j n}}^{k} \alpha_{u n}^{k}}$. With the direct mode subcarrier, the water lever is $\frac{2}{\gamma}$. While with the relayed subcarrier, the water lever is $\frac{1}{\gamma}$.

We now propose the optimal MSSA algorithm. Let us first assume that the optimal transmission mode of each subcarrier is decided. $u_{o p t}^{k}(d)$ is introduced to denote the optimal user allocated for a direct mode subcarrier $k$. $u_{o p t}^{k}(r)$ and $j_{o p t}^{k}(r)$ are introduced to denote the optimal 
user and RS allocated for a relay-aided mode subcarrier $k$. According to the water-filling feature of the PA algorithm, we now have the following:

$$
\begin{aligned}
& u_{o p t}^{k}(d)=\arg \max _{u} \frac{\Lambda_{\mathrm{s}_{n}, \mathrm{~d}_{u n}}^{k, t_{1}}+\Lambda_{\mathrm{s}_{n}, \mathrm{~d}_{u n}}^{k, t_{2}}}{\Lambda_{\mathrm{s}_{n}, \mathrm{~d}_{u n}}^{k, \mathrm{~d}_{1}}} \Lambda_{\mathrm{s}_{n}, \mathrm{~d}_{u n}}^{k,,_{2}}, \forall k \in S_{n}(d), \\
& \left(u_{o p t}^{k}(r), j_{o p t}^{k}(r)\right)=\arg \max _{u, j} \frac{1+\alpha_{u n}^{k}}{\Lambda_{\mathrm{s}_{n}, \mathrm{r}_{j n}}^{k} \alpha_{u n}^{k}}, \forall k \in S_{n}(r) .
\end{aligned}
$$

With $u_{o p t}^{k}(d)$ and $\left(u_{o p t}^{k}(r), j_{o p t}^{k}(r)\right)$ calculated for each subcarrier $k$, we now propose the optimal mode selection method for the subcarrier. Let us define

$$
c_{2, n}^{k}=\frac{\Lambda_{\mathrm{s}_{n}, \mathrm{r}_{j n}}^{k} \alpha_{u^{\prime} n}^{k}}{1+\alpha_{u^{\prime} n}^{k}}-\frac{\Lambda_{\mathrm{s}_{n}, \mathrm{~d}_{u n}}^{k, \mathrm{t}_{1}}+\Lambda_{\mathrm{s}_{n}, \mathrm{~d}_{u n}}^{k, t_{2}}}{2},
$$

where indices $u$ and $\left(u^{\prime}, j\right)$ are assigned with $u_{\text {opt }}^{k}(d)$ and $\left(u_{\text {opt }}^{k}(r), j_{\text {opt }}^{k}(r)\right)$, respectively. When $c_{2, n}^{k}<0$, we certainly have $R_{u n, 1}^{k}>R_{u j n, 2}^{k}$, meaning the direct mode should be chosen for this subcarrier $k$. On the contrary, when $c_{2, n}^{k}>0$, we define

$$
\begin{aligned}
& F_{1}\left(P_{n}^{k}\right)=P_{n}^{k} \frac{\Lambda_{\mathrm{s}_{n}, \mathrm{~d}_{u n}}^{k, t_{1}}+\Lambda_{\mathrm{s}_{n}, \mathrm{~d}_{u n}}^{k, t_{2}}}{2}+\left(P_{n}^{k}\right)^{2} \frac{\Lambda_{\mathrm{s}_{n}, \mathrm{~d}_{u n}}^{k, t_{1}} \Lambda_{\mathrm{s}_{n}, \mathrm{~d}_{u n}}^{k, t_{2}}}{4} \\
& +\frac{\left(\Lambda_{\mathrm{s}_{n}, \mathrm{~d}_{u n}}^{k, t_{1}}-\Lambda_{\mathrm{s}_{n}, \mathrm{~d}_{u n}}^{k, t_{2}}\right)^{2}}{4 \Lambda_{\mathrm{s}_{n}, \mathrm{~d}_{u n}}^{k, t_{1}} \Lambda_{\mathrm{s}_{n}, \mathrm{~d}_{u n}}^{k, t_{2}}}-P_{n}^{k} \frac{\Lambda_{\mathrm{s}_{n}, \mathrm{r}_{j n}}^{k} \alpha_{u^{\prime} n}^{k}}{1+\alpha_{u^{\prime} n}^{k}}
\end{aligned}
$$

If $F_{1}\left(P_{n}^{k}\right)<0$, we certainly have $R_{u n, 1}^{k}<R_{u j n, 2}^{k}$, meaning that the relay-aided mode should be chosen for this subcarrier $k$. Otherwise, we still have $R_{u n, 1}^{k}>R_{u j n, 2}^{k}$, meaning that the direct mode should be chosen for this subcarrier $k$. Let us further denote $F_{1}\left(P_{n}^{k}\right)=\left(P_{n}^{k}\right)^{2} c_{1, n}^{k}-P_{n}^{k} c_{2, n}^{k}+$ $\frac{c_{3, n}^{k}}{4 c_{1, n}^{k}}<0$, where

$$
\begin{aligned}
c_{1, n}^{k} & =\frac{\Lambda_{\mathrm{s}_{n}, \mathrm{~d}_{u n}}^{k, t_{1}} \Lambda_{\mathrm{s}_{n}, \mathrm{~d}_{u n}}^{k, t_{2}}}{4}, \\
c_{3, n}^{k} & =\frac{\left(\Lambda_{\mathrm{s}_{n}, \mathrm{~d}_{u n}}^{k, t_{1}}-\Lambda_{\mathrm{s}_{n}, \mathrm{~d}_{u n}}^{k, \mathrm{~d}_{2}}\right)^{2}}{4} .
\end{aligned}
$$

Here, index $u$ is assigned with $u_{o p t}^{k}(d)$. Note that $F_{1}\left(P_{n}^{k}\right)$ is a quadratic function. $c_{1, n}^{k}>0, c_{2, n}^{k}>0, c_{3, n}^{k}>0$, the discriminant of $F_{1}\left(P_{n}^{k}\right)$ is $\left(c_{2, n}^{k}\right)^{2}-4 c_{1, n}^{k} \frac{c_{3, n}^{k}}{4 c_{1, n}^{k}}=\left(c_{2, n}^{k}\right)^{2}-$ $c_{3, n}^{k}$ and the roots of $F_{1}\left(P_{n}^{k}\right)$ are $\frac{c_{2, n}^{k}+\sqrt{\left(c_{2, n}^{k}\right)^{2}-c_{3, n}^{k}}}{2 c_{1, n}^{k}}>0$ and $\frac{c_{2, n}^{k}-\sqrt{\left(c_{2, n}^{k}\right)^{2}-c_{3, n}^{k}}}{2 c_{1, n}^{k}}>0$. Thus, $F_{1}\left(P_{n}^{k}\right)<0$ is valid only for $\left(c_{2, n}^{k}\right)^{2}>c_{3, n}^{k}$ and

$\frac{c_{2, n}^{k}-\sqrt{\left(c_{2, n}^{k}\right)^{2}-c_{3, n}^{k}}}{2 c_{1, n}^{k}}<P_{n}^{k}<\frac{c_{2, n}^{k}+\sqrt{\left(c_{2, n}^{k}\right)^{2}-c_{3, n}^{k}}}{2 c_{1, n}^{k}}$.

Considering (56) and (58), $\left(c_{2, n}^{k}\right)^{2}>c_{3, n}^{k}$ is equivalent to $c_{4, n}^{k}>c_{5, n}^{k}$, where

$$
\begin{aligned}
c_{4, n}^{k} & =\frac{\Lambda_{\mathrm{s}_{n}, \mathrm{r}_{j n}}^{k}}{1+\alpha_{u_{u^{\prime} n} n}^{k}} \\
c_{5, n}^{k} & =\max \left\{\frac{\Lambda_{\mathrm{s}_{n}, \mathrm{~d}_{u n}}^{k, t_{1}}}{2}, \frac{\Lambda_{\mathrm{s}_{n}, \mathrm{~d}_{u n}}^{k, t_{2}}}{2}\right\} .
\end{aligned}
$$

Here, indices $u$ and $\left(u^{\prime}, j\right)$ are assigned with $u_{o p t}^{k}(d)$ and $\left(u_{\text {opt }}^{k}(r), j_{\text {opt }}^{k}(r)\right)$, respectively.

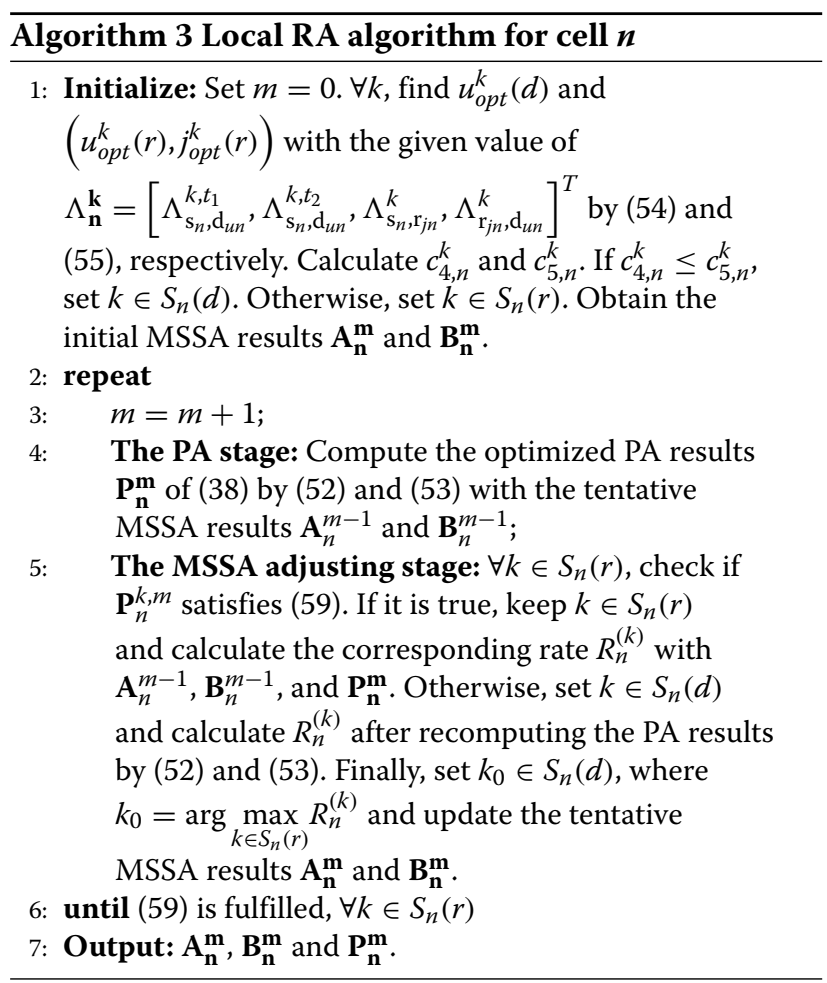

As depicted by Algorithm 3, we now propose an optimal RA algorithm inspired from [16] for each cell $n$ given the value of $\Lambda_{\mathbf{n}}^{\mathbf{k}}=\left[\Lambda_{\mathrm{s}_{n}, \mathrm{~d}_{u n}}^{k, t_{1}}, \Lambda_{\mathrm{s}_{n}, \mathrm{~d}_{u n}}^{k, t_{2}}, \Lambda_{\mathrm{s}_{n}, \mathrm{r}_{j n}}^{k}, \Lambda_{\mathrm{r}_{j n}, \mathrm{~d}_{u n}}^{k}\right]^{T}$. Specifically, we first obtain $u_{o p t}^{k}(d)$ and $\left(u_{o p t}^{k}(r), j_{\text {opt }}^{k}(r)\right)$ by (54) and (55), respectively. Then, we calculate $c_{4, n}^{k}, c_{5, n}^{k}, \forall k$. If $c_{4, n}^{k} \leq c_{5, n}^{k}$, the direct mode should be chosen for the 
subcarrier $k$. Otherwise, the relay-aided mode is still an option and tentatively chosen for this subcarrier $k$. Then, with the tentative MSSA results, we carry out the proposed water-filling PA algorithm, as depicted in (52) and (53). With the tentative PA results, we further check the power criterion (59) for each relayed subcarrier. If (59) is fulfilled, we keep the mode for the relayed subcarrier. Out of all subcarriers violating (59), we only change the mode of the subcarrier $k$ which gives rise to the largest rate increase when switched to the direct mode. The PA algorithm is applied again. This procedure is iterated until none of the relayed subcarrier violates (59).

We now propose the modified IWF algorithm, which consists of two stages as depicted in Algorithm 2. Specifically, we first set $m^{\prime}=0$ and initialize the PA results either by the UPA algorithm or with zeros. During the CCI updating stage of iteration $m^{\prime}, \Lambda_{n}$ of each cell $n$ can be calculated with both the local power $\mathbf{P}_{n}^{\left(m^{\prime}-1\right)}$ and the received CCI power $\mathbf{Q}_{n}^{\left(m^{\prime}-1\right)}$ at each station of the cell. Note that, in practice, the received CCI power values of a cell $n$ can be estimated at each local receiver of the cell. Thus, the CCI updating stage can be carried out autonomously in each cell. During the RA stage, the proposed Algorithm 1 is applied in each cell to calculate its local RA results. The two stages are carried out iteratively until the iteration number $m^{\prime}$ exceeds a prescribed value $M^{\prime}$. Note that, as both stages can be executed autonomously in each cell, the proposed modified IWF algorithm is autonomous. Although it seems intractable to derive conditions under which the modified IWF algorithm provably converges, the convergence is always observed in our experiments. As will be illustrated in "Section 5", the modified IWF algorithm is recommended as it provides a good trade-off between the performance and the complexity.

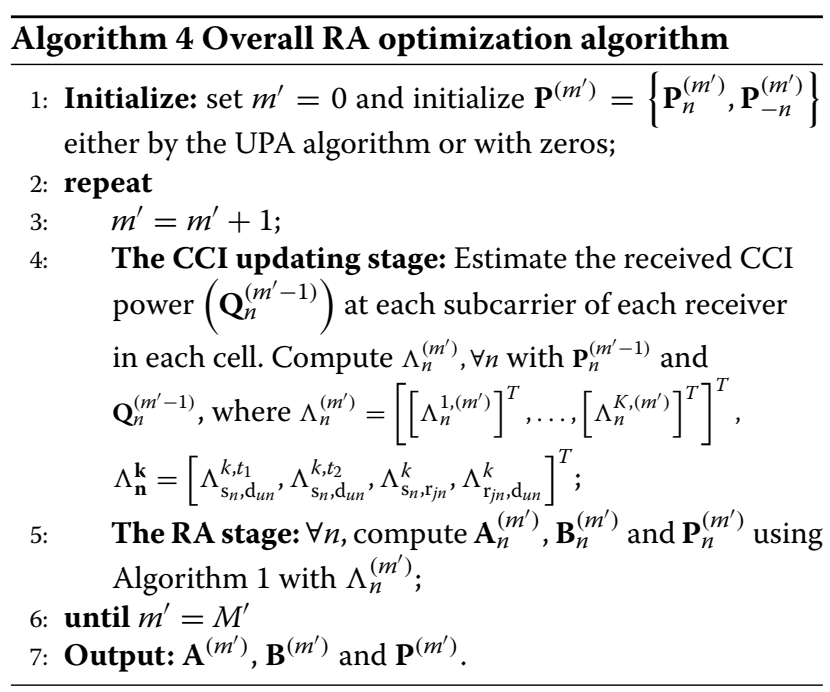

\section{Numerical experiments}

In this section, we evaluate the performances of two proposed RA algorithms. Both the convergence and the effectiveness of the SCLD-based RA algorithm and the modified IWF algorithm will be illustrated. For the convergence, sum rates w.r.t. iterations are first presented with different initialization methods for one particular channel realization. Then for the effectiveness, results averaged over many channel realizations are provided and discussed for the proposed algorithms.

\subsection{System setup}

As shown in Figure 2, a multi-cell OFDMA system with $N=19$ coordinated cells and $K=32$ available subcarriers is considered for illustration. Each cell contains $U=4 \mathrm{MSs}$ and $R=3 \mathrm{RSs}$. Red squares, blue diamonds, and black dots denote the BSs, RSs, and MSs, respectively. An eight-tap delay line model is introduced to randomly generate the channel impulse response (CIR) of each link. Here, each tap obeys a circular complex gaussian distribution with zero-mean and variance as $\sigma_{i}^{2}$. We further assume $\frac{\sigma_{i}^{2}}{\sigma_{i+1}^{2}}=e^{3}$ and $\sum_{i} \sigma_{i}^{2}=d^{-3}$. Finally, we set $P_{T}^{n}=$ $P_{T}, \forall n, \sigma^{2}=-80 \mathrm{dBm}, \lambda_{0}=K, \mu_{0}=0.5, \alpha_{\lambda}=K / 4$, $\alpha_{\mu}=0.02, \epsilon_{1}=R_{\text {ini }} / 500, \epsilon_{2}=1, \epsilon_{3}=R_{\text {ini }} / 500$, and $M^{\prime}=15$. Here, $R_{i n i}$ denotes the sum rate calculated with the initial RA results at the beginning of each iteration.

\subsection{Results for the SCLD-based RA algorithm with three initialization methods}

For the proposed SCLD-based RA algorithm, three initialization PA algorithms (RPA, UPA, and BPA) are introduced. Specifically, RPA corresponds to the random power allocation. Each power value of each subcarrier is randomly drawn from a uniform distribution in $[0,1]$ and normalized to achieve a total power value equal to $P_{T}$. UPA corresponds to the uniform power allocation algorithm, and BPA corresponds to applying the optimal PA algorithm proposed in [16] when the $\mathrm{CCI}$ is always set to 0 .

In order to compare the performance of the proposed SCLD-based RA algorithm using three initialization methods, we set $N=5$ and generate 200 random realizations of channels. Different total power constraints are set up ranging from 0 to $50 \mathrm{dBm}$. The three PA algorithms (RPA, UPA, and BPA) are applied to initialize the proposed SCLD-based RA algorithm. As shown in Figure 3 , the average sum rates are similar with the three initialization algorithms under all power constraints. The execution time with BPA however is much lower than those with UPA and RPA.

We further generate three realizations of channels, each of which corresponds to a specific total power constraint $(0 / 20 / 40 \mathrm{dBm})$. For each channel realization, we randomly 


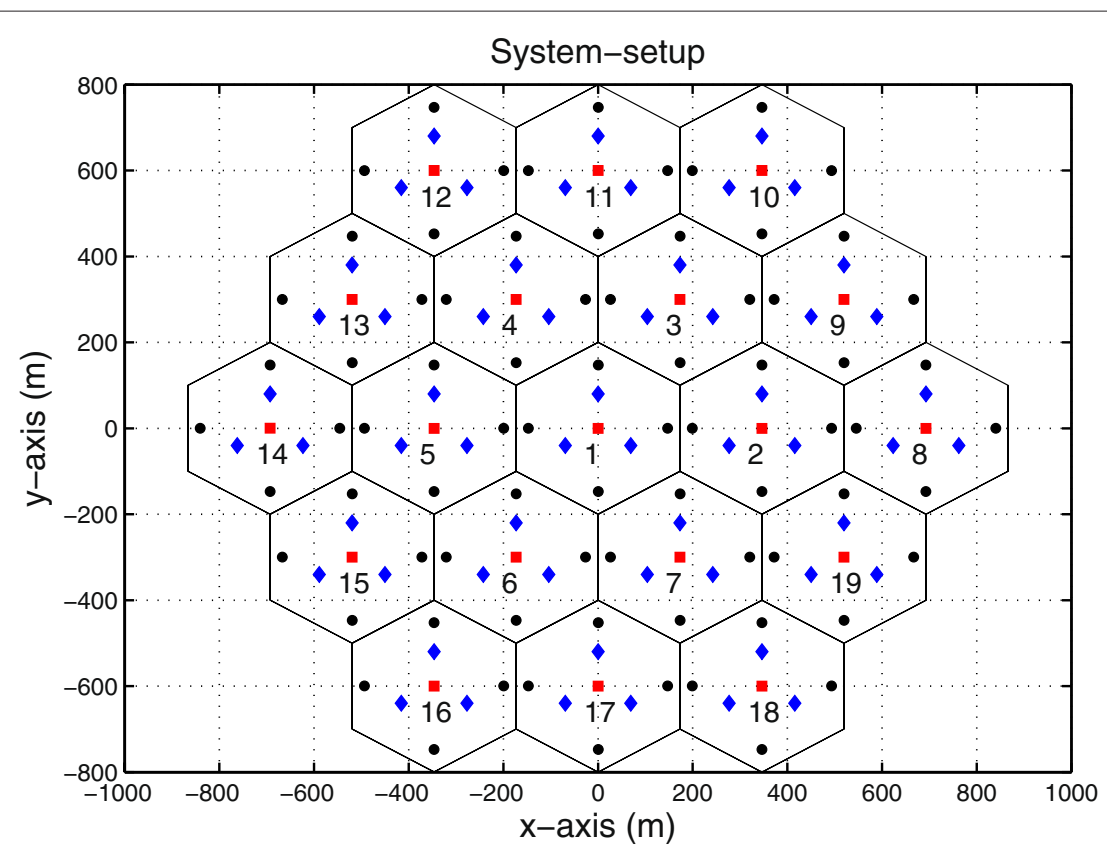

Figure 2 A random system setup.

(a)

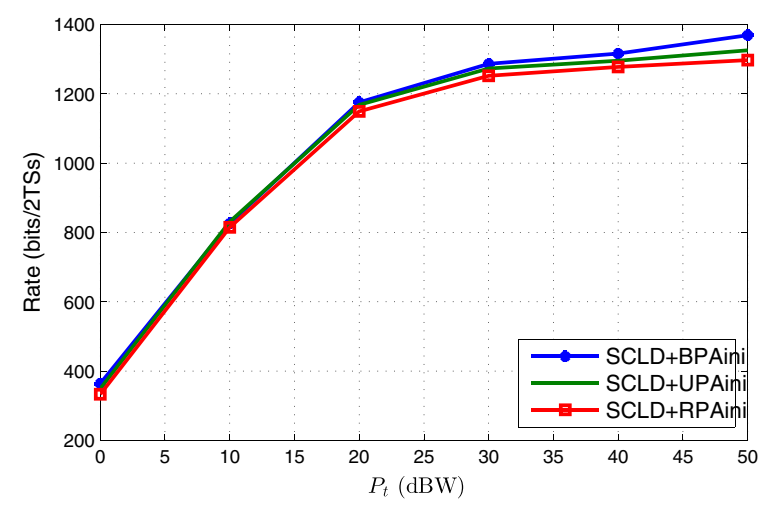

(b)

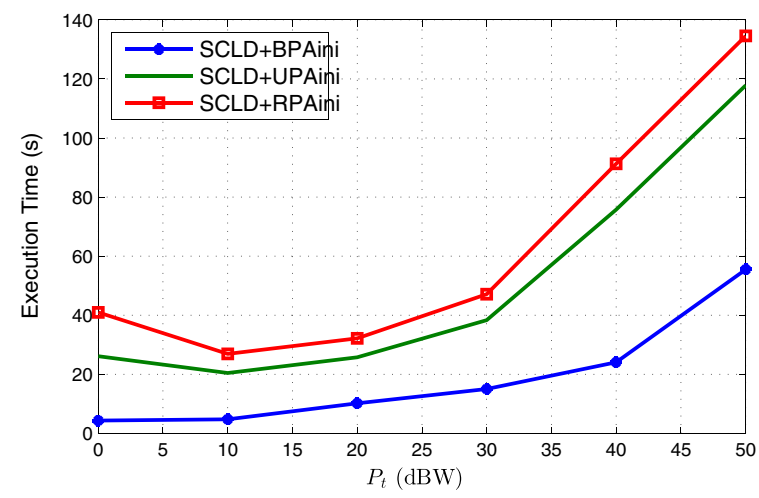

Figure 3 Average performances w.r.t. different power constraints with five cells. (a) Average sum rates. (b) Average execution time. generate 500 initial points using the RPA algorithm and compare the performance with those obtained when the initial points are generated by the UPA and the BPA. As shown in Figure 4, the average sum rates are similar with the three initialization algorithms under all power constraints. The execution time with BPA however is much lower than those with UPA and RPA.

Thus, in the following simulations, we will initialize the proposed SCLD-based RA algorithm with BPA.

\subsection{Results for a random realization of channels}

In order to illustrate the convergence of our proposed algorithms, we set $N=10$ and randomly generate a set of channels when $P_{T}=40 \mathrm{dBm}$. As shown in Figure 5, for the proposed SCLD-based RA algorithm, the total rates keep increasing continuously and converge smoothly to the final rate. For the modified IWF algorithm, the total rates keep increasing with slight fluctuations. After convergence, the total rate is increased by around $37 \%, 34 \%$, and 27\% using the CA in [25], the SCLD-based RA algorithm, and the modified IWF algorithm, respectively. Note that the modified IWF algorithm converges very fast. As will be further illustrated, the modified IWF algorithm with one step outer iteration performs similarly as the modified IWF algorithm with 15 iterations.

\subsection{Results averaged over channel distribution}

To illustrate the effectiveness of the proposed two RA algorithms, we set $N=3$ and generate 100 random realizations of channels. Different total power constraints are 


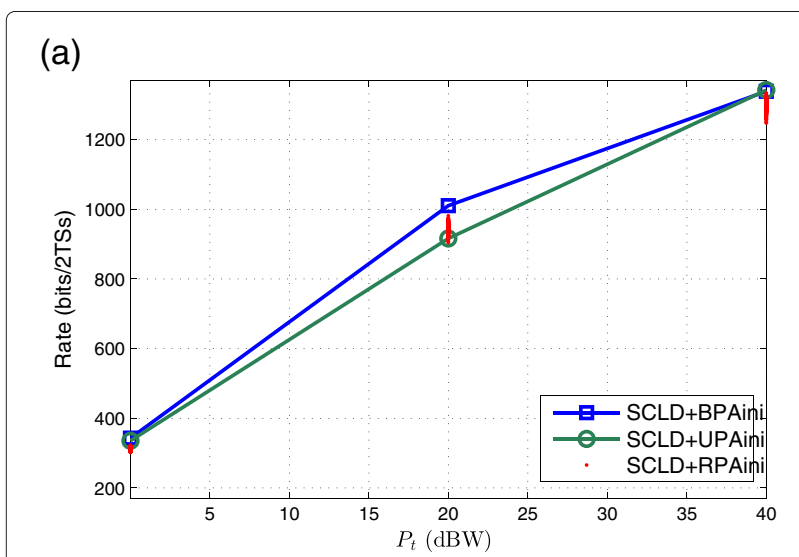

(b)

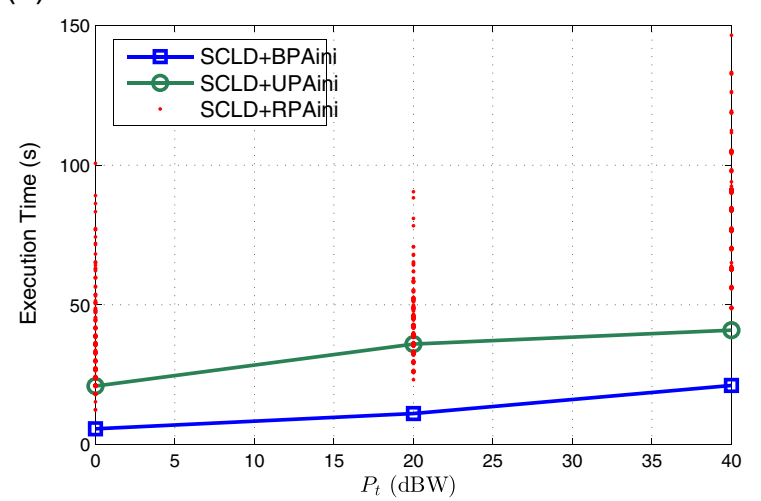

Figure 4 Average performances w.r.t. different initial points with five cells. (a) Average sum rates. (b) Average execution time.

set up ranging from 0 to $50 \mathrm{dBm}$. For the purpose of comparison, we introduce two benchmark algorithms (BAs). Here, BA1 corresponds to the optimal RA algorithm proposed in [16], when the CCI is always set to 0. BA2 corresponds to the algorithm when the power is uniformly

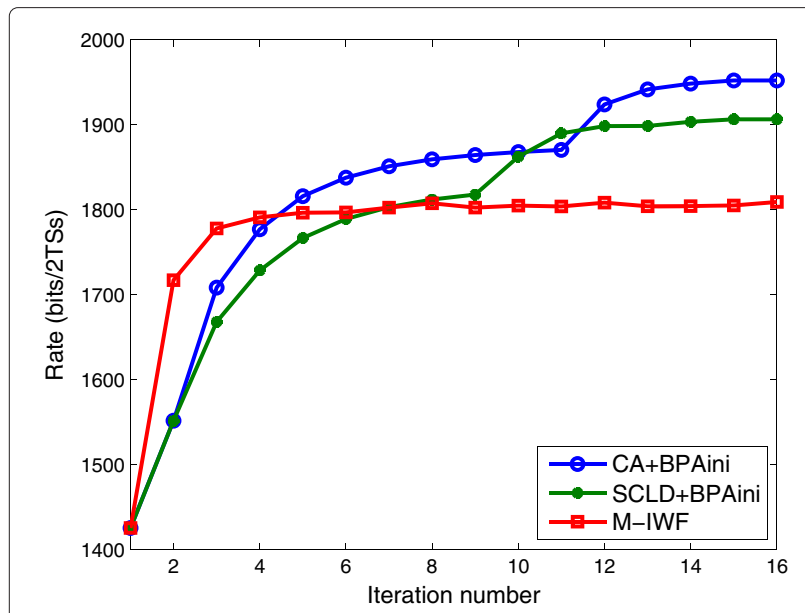

Figure 5 Rate results for the random realization of channels. allocated, and the RS and MS are randomly selected for each subcarrier.

As shown in Figure 6a, the two proposed algorithms outperform the two benchmark algorithms. Note that, at low SNR scenarios, both the modified IWF algorithm and BA1 performs similarly as our proposed algorithms. This is because both the modified IWF algorithm and BA1 are near-optimal when the $\mathrm{CCI}$ is small. The sum rate of the proposed SCLD-based RA algorithm is close to that of the CA, especially at high SNR scenarios.

We further set $N=10$ and generate 100 random realizations of channels. As shown in Figure $6 \mathrm{~b}$, the same conclusion can be found as that shown in Figure 6a. Thus, the effectiveness of the proposed RA algorithms is illustrated.

To illustrate the complexity and effectiveness of the proposed two RA algorithms, we set $P_{T}=40 \mathrm{dBm}$ and

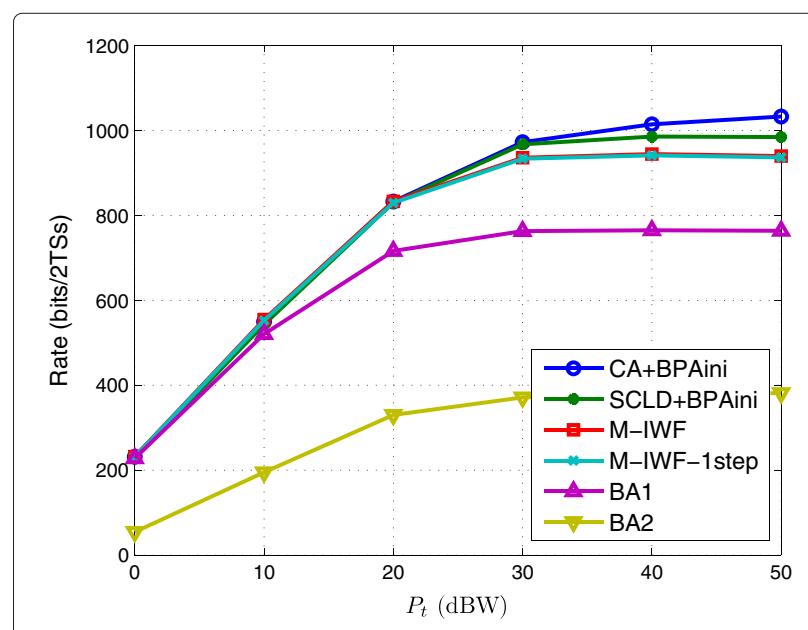

(a) 3 cell scenario

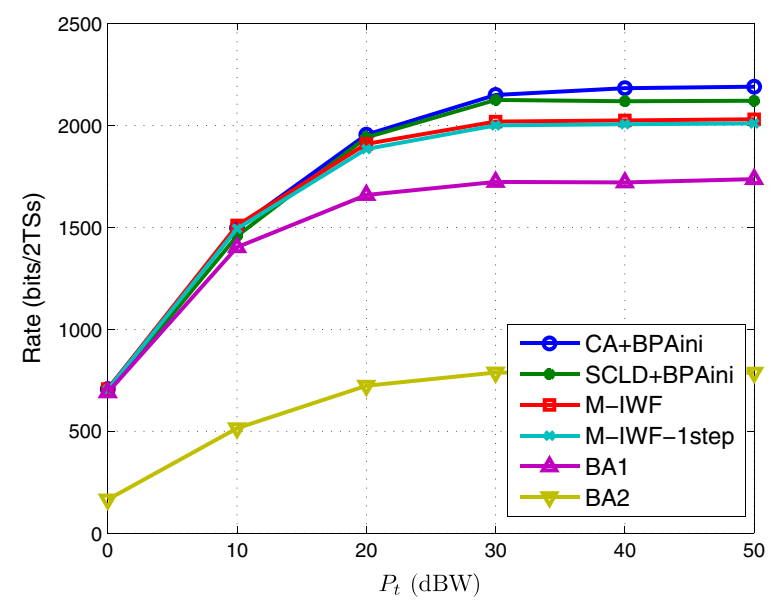

(b) 10 cell scenario

Figure 6 Average performances w.r.t. different power constraints. (a) three-cell scenario. (b) ten-cell scenario. 
generate a series of system setups with different cell numbers ranging from 2 to 18 . For each system setup, average execution times and performances of the CA in [25], the SCLD-based RA algorithm and the modified IWF algorithm are presented over 20 random realizations of channels.

As shown in Figure 7, both the SCLD-based RA algorithm and the modified IWF algorithm outperform the BAs, especially when the cell number is quite large. The SCLD-based RA algorithm results in sum rates similar to those obtained with the CA, while its execution time is much less than that of the centralized one. Thus, the SCLD-based RA algorithm provides good trade-off between performance and complexity, meaning that it is much more practical than the centralized one.

Also, the modified IWF algorithm results in sum rates close to those obtained with the SCLD-based RA

(a)

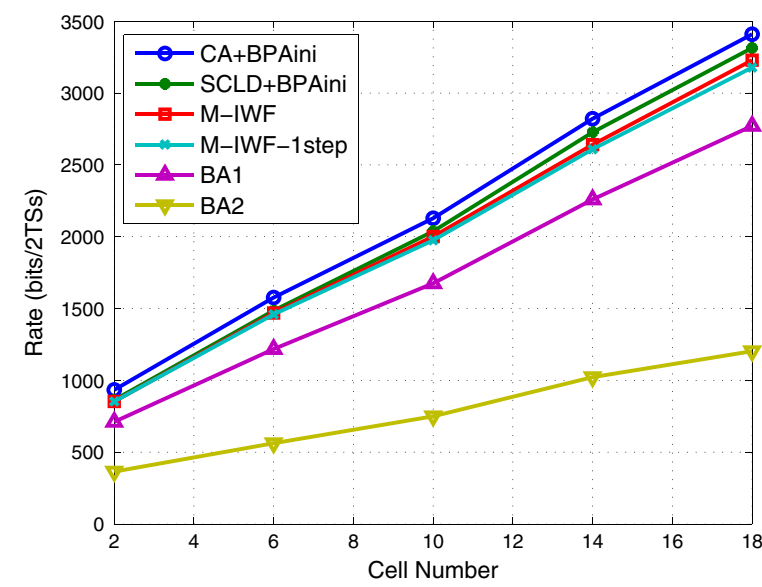

(b)

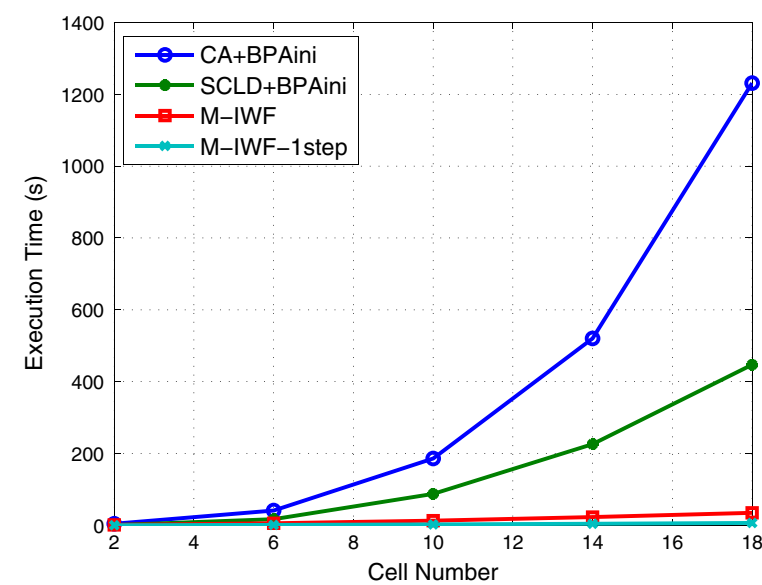

Figure 7 Average performances w.r.t. different cell numbers when $\boldsymbol{P}_{\boldsymbol{T}}=\mathbf{4 0} \mathrm{dBm}$. (a) Average sum rates. (b) Average execution time. algorithm, while it is autonomous and consumes less execution time than the SCLD-based RA algorithm. Thus, the modified IWF algorithm is more preferred for practical implementation. Note that the convergence of the modified IWF algorithm is always found in simulations, although it is not theoretically guaranteed. More interestingly, the modified IWF algorithm with one step outer iteration performs similarly as the modified IWF algorithm with 15 iterations.

We further set $P_{T}=5 \mathrm{dBm}$. As shown in Figure 8, both the SCLD-based RA algorithm and the modified IWF algorithm perform similarly as the CA and slightly better than the BA1. This is because both the modified IWF algorithm and BA 1 are near-optimal when the CCI is small. Note that the modified IWF algorithm is autonomous and less complex than the SCLD-based RA algorithm. Thus, it is recommended for practical implementation.

(a)

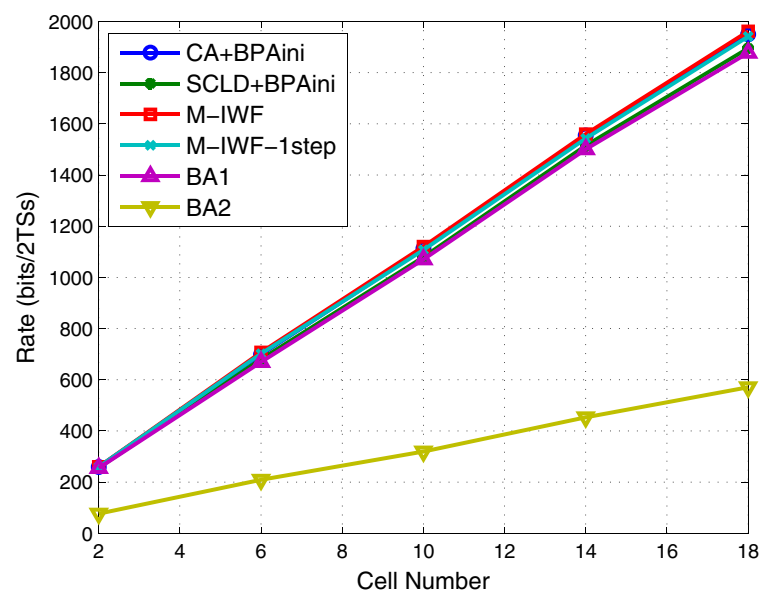

(b)

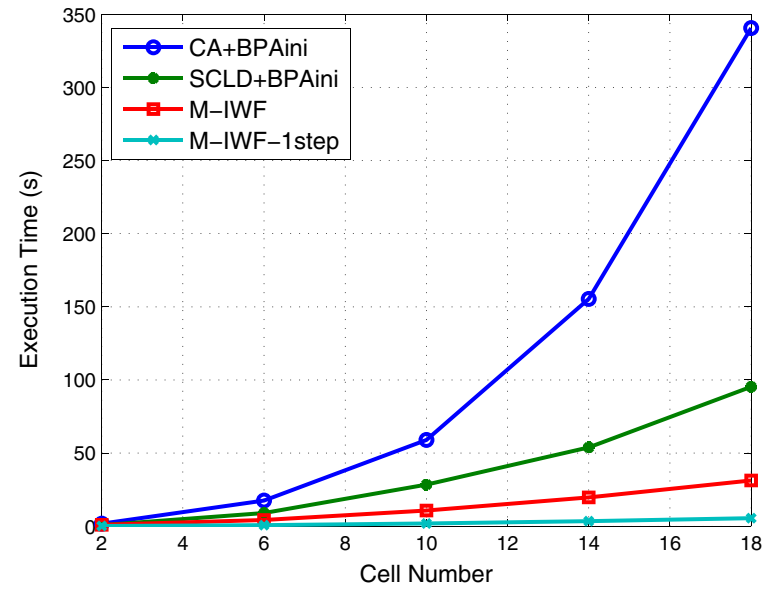

Figure 8 Average performances w.r.t. different cell numbers when $\boldsymbol{P}_{\boldsymbol{T}}=\mathbf{5} \mathbf{d B m}$. (a) Average sum rates. (b) Average execution time. 


\section{Conclusions}

We have considered a multi-cell OFDMA downlink system with several DF and RSs aiding the BS transmissions. An iterative SCLD-based RA algorithm and a modified IWF algorithm have been proposed to solve the sum rate maximization problem with per cell total power constraints. Specifically, the iterative SCLD-based RA algorithm was semi-distributed with a central controller for message passing and the modified IWF algorithm was autonomous. Through numerical experiments, we have illustrated that the two proposed algorithms converge fast and are time saving compared to CA.

\section{Competing interests}

The authors declare that they have no competing interests.

\section{Acknowledgements}

The authors would like to thank Prof. S. Boyd for sharing the GGPLAB toolbox online, China Scholarship Council (CSC) for funding this work, and the Belgian Science Policy Office (BELSPO) for the support of the Inter-university Attractive Poles Programme (IAP) BESTCOM network. The work of Tao Wang is supported by The Program for Professor of Special Appointment (Eastern Scholar) at the Shanghai Institutions of Higher Learning, Innovation Program of Shanghai Municipal Education Commission (14ZZ096), Innovation Fund of Shanghai University, and SRFDP (20133108120015).

\section{Author details}

${ }^{1}$ ICTEAM institute (École Polytechnique de Louvain), Université catholique de Louvain, Place du Levant, 1348 Louvain-la-Neuve, Belgium. ${ }^{2}$ School of Communication and Information Engineering, Shanghai University, 99 Shangda Rd, Baoshan, 200072 Shanghai, China. ${ }^{3}$ School of Electronic Science and Engineering, National University of Defense Technology, 47 Yan Wachi Street, 410073 Changsha, China.

Received: 12 March 2014 Accepted: 11 September 2014 Published: 23 September 2014

\section{References}

1. R Pabst, BH Walke, DC Schultz, P Herhold, H Yanikomeroglu, S Mukherjee, H Viswanathan, M Lott, W Zirwas, M Dohler, H Aghvami, DD Falconer, GP Fettweis, Relay-based deployment concepts for wireless and mobile broadband radio. IEEE Commun. Mag. 42(9), 80-89 (2004)

2. JN Laneman, GW Wornell, Distributed space-time-coded protocols for exploiting cooperative diversity in wireless networks. IEEE Trans. Inform. Theory. 49(10), 2415-2425 (2003)

3. JN Laneman, DNC Tse, GW Wornell, Cooperative diversity in wireless networks: Efficient protocols and outage behavior. IEEE Trans. Inform. Theory. 50(12), 3062-3080 (2004)

4. W Wang, S Yan, S Yang, Optimally joint subcarrier matching and power allocation in OFDM multihop system. Eurasip J. Adv. Signal Process. 2008, 1-8 (2008)

5. W Wang, R Wu, Capacity maximization for OFDM two-hop relay system with separate power constraints. IEEE Trans. Vehicular Technol. 58(9), 4943-4954 (2009)

6. Y Li, W Wang, J Kong, M Peng, Subcarrier pairing for amplify-and-forward and decode-and-forward OFDM relay links. IEEE Commun. Lett. 13(4), 209-211 (2009)

7. TWang, Weighted sum power minimisation for multichannel decode-and-forward relaying. IET Electron. Lett. 48(7), 410-411 (2012)

8. L Vandendorpe, RT Duran, J Louveaux, A Zaidi, Power allocation for OFDM transmission with DF relaying. Paper presented at IEEE International Conference on Communications (Beijing, 19-23 May 2008), pp. 3795-3800

9. J Louveaux, RT Duran, L Vandendorpe, Efficient algorithm for optimal power allocation in OFDM transmission with relaying. Paper presented as IEEE international conference on acoustics, speech and signal processing (Dublin, 22-25 April 2007), pp. 3257-3260
10. TCY Ng, W Yu, Joint optimization of relay strategies and resource allocations in cooperative cellular networks. IEEE J. Select. Areas Commun. 25(2), 328-339 (2007)

11. W Ying, Q Xin-chun, W Tong, L Bao-ling, Power allocation and subcarrier pairing algorithm for regenerative OFDM relay system. Paper presented at IEEE 65th vehicular technology conference (Dublin, 22-25 April 2007), pp. 2727-2731

12. Y Li, W Wang, J Kong, W Hong, X Zhang, M Peng, Power allocation and subcarrier pairing in OFDM-based relaying networks. Paper presented at IEEE international conference on communications (Beijing, 19-23 May 2008), pp. 2602-2606

13. M Hajiaghayi, M Dong, B Liang, Optimal channel assignment and power allocation for dual-hop multi-channel multi-user relaying. Paper presented at IEEE INFOCOM (Shanghai, 10-15 April 2011), pp. 76-80

14. L Vandendorpe, J Louveaux, O Oguz, A Zaidi, Improved OFDM transmission with DF relaying and power allocation for a sum power constraint. Paper presented at 3rd international symposium on wireless pervasive computing (Santorini, 7-9 May 2008), pp. 665-669

15. L Vandendorpe, J Louveaux, O Oguz, A Zaidi, Power allocation for improved DF relayed OFDM transmission: the individual power constraint case. Paper presented at IEEE international conference on communications (Dresden, 14-18 June 2009), pp. 1-6

16. L Vandendorpe, J Louveaux, O Oguz, A Zaidi, Rate-optimized power allocation for DF-relayed OFDM transmission under sum and individual power constraints. EURASIP J. Wireless Commun. Network. 2009(11) (2009). doi:10.1155/2009/814278

17. C-N Hsu, H-J Su, P-H Lin, Joint subcarrier pairing and power allocation for OFDM transmission with decode-and-forward relaying. IEEE Trans. Signal Process. 59(1), 399-414 (2011)

18. TWang, L Vandendorpe, Wsr maximized resource allocation in multiple DF relays aided OFDMA downlink transmission. IEEE Trans. Signal Process. 59(8), 3964-3976 (2011)

19. TWang, L Vandendorpe, Sum rate maximized resource allocation in multiple DF relays aided OFDM transmission. IEEE J. Selected Areas Commun. 29(8), 1559-1571 (2011, September)

20. H Boostanimehr, VK Bhargava, Selective subcarrier pairing and power allocation for DF OFDM relay systems with perfect and partial csi. IEEE Trans. Wireless Commun. 10(12), 4057-4067 (2011, December)

21. Y Liu, M Tao, An optimal graph approach for optimizing OFDMA relay networks. Paper presented at 2012 IEEE international conference on communications (Ottawa, Ontario, 10), pp. 4277-4281

22. TWang, Y Fang, L Vandendorpe, Power minimization for OFDM transmission with subcarrier-pair based opportunistic DF relaying. IEEE Commun. Lett. 17(3), 471-474 (2013, March)

23. TWang, F Glineur, J Louveaux, L Vandendorpe, Weighted sum rate maximization for downlink OFDMA with subcarrier-pair based opportunistic DF relaying. IEEE Trans. Signal Process. PP(99), 1-1 (2013)

24. N Himayat, S Talwar, A Rao, R Soni, Interference management for $4 \mathrm{~g}$ cellular standards [wimax/Ite update]. IEEE Commun. Mag. 48(8), 86-92 (2010)

25. Z Jin, L Vandendorpe, Resource allocation in multi-cellular DF relayed OFDMA systems. Paper presented at IEEE global telecommunications conference (Houston, Texas, 5-9 Dec 2011), pp. 1-5

26. Z Jin, T Wang, J-B Wei, L Vandendorpe, Resource allocation for maximizing weighted sum of per cell min-rate in multi-cell DF relay aided downlink OFDMA systems. Paper presented at 2012 IEEE 23rd international symposium on personal indoor and mobile radio communications (Sydney, New South Wales, 9-12 Sept 2012), pp. $1845-1850$

27. M Salem, A Adinoyi, H Yanikomeroglu, D Falconer, Opportunities and challenges in OFDMA-based cellular relay networks A radio resource management perspective. IEEE Trans. Vehic. Technol. 59(5), 2496-2510 (2010)

28. M Salem, A Adinoyi, M Rahman, H Yanikomeroglu, D Falconer, Y-D Kim, E Kim, Y-C Cheong, An overview of radio resource management in relay-enhanced OFDMA-based networks. IEEE Commun. Surv. Tutor 12(3), 422-438 (2010)

29. L Venturino, N Prasad, X Wang, Coordinated scheduling and power allocation in downlink multicell OFDMA networks. IEEE Trans. Vehic. Technol. 58(6), 2835-2848 (2009) 
30. M Pischella, J-C Belfiore, Weighted sum throughput maximization in multicell OFDMA networks. IEEE Trans. Vehic. Technol. 59(2), 896-905 (2010)

31. T Wang, L Vandendorpe, Iterative resource allocation for maximizing weighted sum min-rate in downlink cellular OFDMA systems. IEEE Trans. Signal Process. 59(1), 223-234 (2011)

32. H Zhang, L Venturino, N Prasad, P Li, S Rangarajan, X Wang, Weighted sum-rate maximization in multi-cell networks via coordinated scheduling and discrete power control. IEEE J. Selected Areas Commun. 29(6), 1214-1224 (2011)

33. M Chiang, CW Tan, DP Palomar, DO'Neill, D Julian, Power control by geometric programming. IEEE Trans. Wireless Commun. 6(7), 2640-2651 (2007)

34. BR Marks, GP Wright, A general inner approximation algorithm for nonconvex mathematical programs. Oper. Res. 26(4), 681-683 (July/August 1978)

35. RD Yates, A framework for uplink power control in cellular radio systems IEEE J. Selected Areas Commun. 13(7), 1341-1347 (1995)

36. W Yu, G Ginis, JM Cioffi, Distributed multiuser power control for digital subscriber lines. Selected Areas Commun. IEEE J. 20(5), 1105-1115 (2002)

doi:10.1186/1687-1499-2014-154

Cite this article as: Jin et al.: Distributed algorithms for sum rate maximization in multi-cell downlink OFDMA with opportunistic DF relaying. EURASIP Journal on Wireless Communications and Networking 2014 2014:154

\section{Submit your manuscript to a SpringerOpen ${ }^{\circ}$ journal and benefit from:}

- Convenient online submission

- Rigorous peer review

- Immediate publication on acceptance

- Open access: articles freely available online

- High visibility within the field

- Retaining the copyright to your article

Submit your next manuscript at $>$ springeropen.com 